J. DIFFERENTIAL GEOMETRY

63 (2003) 475-520

\title{
LOCAL ISOMETRIC EMBEDDING OF SURFACES WITH NONPOSITIVE GAUSSIAN CURVATURE
}

\author{
QING HAN, JIA-XING HONG \& CHANG-SHOU LIN
}

\begin{abstract}
In this paper, we prove the existence of an isometric embedding near the origin in $\mathbf{R}^{\mathbf{3}}$ of a two-dimensional metric with nonpositive Gaussian curvature. The Gaussian curvature can be allowed to be highly degenerate near the origin. Through the Gauss-Codazzi equations, the embedding problem is reduced to a $2 \times 2$ system of the first order derivaties and is solved via the method of Nash-Moser-Hörmander iterative scheme.
\end{abstract}

\section{Introduction}

Given a smooth $n$-dimensional Riemannian manifold $\left(M_{n}, g\right)$, can we find its smooth isometric embedding in the Euclidean space $\mathbf{R}^{\mathbf{p}}$ ? This is a long standing problem in Differential Geometry. As is well-known, the necessary condition is $p \geq s_{n}=n(n+1) / 2$. For this problem there are two aspects. One is global and another is local. The result on the existence of global smooth isometric embedding is first due to Nash in [14]. For a simplified proof and an improved result, see [3]. For the local embedding, we are interested only in the case $p=s_{n}$. The first general result was obtained by Janet and Cartan.

Janet-Cartan Theorem. Any analytic n-dimensional Riemannian manifold always has a local analytic isometric embedding in $\mathbf{R}^{\mathbf{S}_{\mathbf{n}}}$.

The smooth case remains open in general. The following conjecture was posed by Schlaefli in 1873, and also recently reposed by Yau in

The first author is supported in part by an NSF grant and a Sloan research fellowship. The second author is supported in part by the project of MOST of China.

Received 03/14/2003. 
[17] and [18]: any smooth surface always has a local smooth isometric embedding in $\mathbf{R}^{\mathbf{3}}$.

Suppose a metric $g$ defined in an open set $U \subset \mathbf{R}^{2}$ is given by

$$
g=\sum_{i, j=1}^{2} g_{i j} d x_{i} d x_{j}
$$

Isometrically immersing $g$ in $\mathbf{R}^{\mathbf{3}}$ is equivalent to finding a function $\mathbf{r}=$ $\left(X_{1}, X_{2}, X_{3}\right): U \rightarrow \mathbf{R}^{\mathbf{3}}$ such that

$$
d X_{1}^{2}+d X_{2}^{2}+d X_{3}^{2}=g
$$

or

$$
\sum_{\alpha=1}^{3} \frac{\partial X_{\alpha}}{d x_{i}} \cdot \frac{\partial X_{\alpha}}{d x_{j}}=g_{i j}, \quad i, j=1,2 .
$$

This is a first order differential system of three equations for three unknown functions. However, such a system cannot be covered by the general theory of first order differential systems. In order to study this system, we change it to an equivalent differential equation. By a straightforward calculation, one can check that the solvability of (1.1) is equivalent to the solvability of the following equation:

$$
\operatorname{det}\left(\nabla^{2} u\right)=K \operatorname{det}\left(g_{i j}\right)\left(1-|\nabla u|^{2}\right),
$$

with $|\nabla u|<1$, where $\nabla$ denotes the covariant derivatives with respect to $g$ and $K$ is the Gaussian curvature. Then (1.2) can be written as

$$
\operatorname{det}\left(u_{i j}-\Gamma_{i j}^{k} u_{k}\right)=K \operatorname{det}\left(g_{i j}\right)\left(1-g^{i j} u_{i} u_{j}\right)
$$

where $\Gamma_{i j}^{k}$ is the Christoffel symbol and $\left(g^{i j}\right)$ is the inverse of $\left(g_{i j}\right)$.

Equation (1.2) is called the Darboux equation. It is a fully nonlinear equation of the Monge-Ampère type. We are interested in a local solution in a neighborhood of any given point $p \in U$. The type of Equation (1.2) is determined by the sign of the Guassian curvature $K$. It is elliptic if $K$ is positive, and hyperbolic if $K$ negative. It is degenerate if $K$ vanishes.

In the case that the Gaussian curvature $K$ does not vanish at $0 \in U$, (1.2) can be solved easily in a neighborhood of 0 . The difficulty arises if $K$ vanishes. In this direction, Lin made an important breakthrough 
in [8] and [9]. By a delicate analysis, he obtained the existence of local solution of (1.2) and hence the sufficiently smooth isometric embedding for the following two cases: $K(0)=0$ and $d K(0) \neq 0$, or $K(0)=0$ and $K$ nonnegative in a neighborhood of the point 0 . Later on, Nakamura [12] proved the local isometric embedding if $K(0)=0, d K(0)=0$ and $\operatorname{Hess} K(0)<0$. Evidently, $K$ is nonpositive near point 0 and the leading part of $K$ is an irreducible quadratic polynomial. For the case of nonpositive Gaussian curvature, Hong [5] also proved the local isometric embedding in a neighborhood of 0 if $K=h g^{2 m}$, where $h$ is a negative function and $g$ is a function with $g(0)=0$ and $d g(0) \neq 0$. All these results are based on the careful study of (1.2).

In the present paper, we shall study the local isometric embedding of metrics with nonpositive curvature. We first present the main result.

We make the following assumption:

(A) For some positive integer $l$, some small constant $\delta>0$ and some coordinate system $(x, t)$ around the origin, there is a neighborhood $N$ of 0 , such that the following holds: for any $C^{1}$ function $u$ with $|u|_{C^{1}(N)}<\delta, N$ is separated into finitely many subdomains by $C^{1}$ curves $\gamma_{i}: t=t_{i}(x), i=1, \ldots, l$, with $t_{i}(0)=0, t_{i}^{\prime}(0) \neq t_{j}^{\prime}(0)$ for $i \neq j$, and the function $\partial_{t} K+u \partial_{x} K$ changes its sign only across $\gamma_{1}, \ldots, \gamma_{l}$. Moreover, none of the curves $\gamma_{i}, i=1, \ldots, l$, is tangent to the $t$-axis or $x$-axis.

Although the assumption (A) looks complicated, it has a simple geometric interpretation. It means that the zero set of $\partial_{t} K$ consists of finitely many curves meeting nontangentially at the origin and that such a picture is preserved under the perturbation of the directional derivatives of $K$. As we will show in Section 7, the assumption (A) covers a large class of Gaussian curvature, including the cases in [12] and $[5]$.

The main result we will prove is the following:

Theorem 1.1. Suppose a given $C^{r}$ metric g, with $K \leq 0$, satisfies (A) for some $r>2 l+12$. Then $g$ admits a $C^{r-l-6}$ local isometric embedding in $\mathbf{R}^{3}$.

Now we present two results which are the special cases of Theorem 1.1. 
Theorem 1.2. Suppose $g$ is a $C^{r}$ metric whose Gaussian curvature $K$ is nonpositive and given in a neighborhood of 0 by

$$
K=-\left(\nu_{1} \cdot(x, t)\right)^{2} \cdots\left(\nu_{k} \cdot(x, t)\right)^{2} Q(x, t)+\text { h.o.t. }
$$

where $Q(x, t)$ is a homogeneous polynomial of degree $l-2 k$ with $Q(x, t)>$ 0 for $(x, t) \neq 0$ and $\nu_{1}, \ldots, \nu_{k}$ are distinct unit vectors. If $r>2 l+12$, then $g$ admits a $C^{r-l-6}$ local isometric embedding in $\mathbf{R}^{3}$.

An important case in (1.3) is given when the square of the linear functions are not present. In other words, $K$ is given by

$$
K=-Q(x, t)+\text { h.o.t. }
$$

where $Q(x, t)$ is a homogeneous polynomial of degree $l$ with $Q(x, t)>0$ for $(x, t) \neq 0$. Another special case is given if the leading polynomial is quadratic.

Theorem 1.3. Suppose $g$ is a $C^{r}$ metric whose Gaussian curvature $K$ is given in a neighborhood of 0 by

$$
K=h(x, t) Q(x, t) \prod_{j=1}^{k}\left(\nu_{j} \cdot(x, t)-c_{j}(x, t)\right)^{2 n_{j}}
$$

where $h$ is a function with $h(0)<0, Q(x, t)$ is homogeneous polynomial of degree $l-2 k$ with $Q(x, t)>0$ for $(x, t) \neq 0, n_{1}, \ldots, n_{k}$ are positive integers, $\nu_{1}, \ldots, \nu_{k}$ are distinct unit vectors and $c_{1}, \ldots, c_{k}$ are $C^{1}$ functions with $c_{j}(0)=0$ and $\nabla c_{j}(0)=0$. If $r>2 l+12$, then $g$ admits a $C^{r-l-6}$ local isometric embedding in $\mathbf{R}^{3}$.

For a nonpositive function $K$, its least vanishing order is two. The condition (1.3) means that the leading polynomial of $K$ vanishes with the least order along its zero set. In this case, the leading polynomial is decisive and the higher order term does not play any role. If the leading polynomial of $K$ vanishes at the higher order, we need more precise description of $K$ itself. The condition (1.4) implies in particular that $K$ vanishes at the same order along each curve in its zero set except at the origin. If $n_{1}=\cdots=n_{k}=1$ in (1.4), it reduces to (1.3).

Now we describe our method to prove Theorem 1.1, which is different from all methods employed before. We begin with Gauss-Codazzi system, instead of Darboux equation (1.2). The Fundamental Theorem of Surface Theory reduces an embedding of an abstract surface to 
the construction of a smooth solution of the Gauss-Codazzi system. If the Gaussian curvature is negative, a differential system equivalent to Gauss-Codazzi system was derived first by Rozhdestvenskiur [16] when the metric is in the geodesic form, and then by Poznyak in the general case. This system is quasilinear of the hyperbolic type, whose characteristics are the integral curves defined by simple ordinary differential equations. However, such a system cannot be employed directly in our study because the Gaussian curvature is allowed to vanish. To overcome this, we change the Rozhdestvenskil-Poznyak system into another equivalent system, with no restriction on the Gaussian curvatures. In fact the new system can be derived directly from the Gauss-Codazzi system. We shall do so in the present paper. We mention the Rozhdestvenskil-Poznyak system because its simple characterization of the characteristics is the basis for our a priori estimates. For discussions of the RozhdestvenskiuPoznyak system and its applications, see [15] and references there.

The new system is a $2 \times 2$ quasilinear differential system of the first order in $\mathbf{R}^{\mathbf{2}}=\{(\mathbf{x}, \mathbf{t})\}$. In order to solve this system, we need to study its linearized system. The linearized system is hyperbolic if Gaussian curvature $K$ is nonpositive, and is degenerate while $K$ vanishes. So far, there is no general result on the solvability of the Cauchy problem for the degenerate hyperbolic systems. We have to take advantage of the special features of our system. In general, an efficient method to study first order differential systems is to symmetrize the system and then attempt to derive energy estimates, as in [2]. However, symmetrization would destroy the special feature of our system. In our case, an essential quantity is the derivative of Guassian curvature $K$ in the time direction, i.e., $\partial_{t} K$. In the region where the time derivative is positive, a priori estimates for the linearized equation can be obtained by relating this system to the linearized equation of the Rozhdestvenskil-Poznyak system, for which we can follow the characteristic curves easily. In fact we compare our solution with a solution to a single differential equation and the difference is the solution to the Rozhdestvenskil-Poznyak system. The case when the time derivative is negative is tricky. If we follow a similar process for the case of positive time derivative, we can only get estimates on one component of solutions, which are vectors of two components. To continue, we need to differentiate the linearized equation and study the resulted equation satisfied by the derivatives of solutions. It turns out this equation has the same property of the original equation. Now we have an estimate on one component of solutions and its derivatives. From this extra information on the derivatives of 
one component, we can get estimates on another component (itself). Last we need the assumption (A) to put all these estimates together.

It would be desirable that the condition (A) be removed. We are unable to achieve this in the present paper. We should mention that in general a degenerate hyperbolic system may have no local solutions. For such an example, see Egorov [1].

Recently, Nadirashvili [10] constructed a smooth metric in the unit disc $B_{1} \subset \mathbf{R}^{\mathbf{2}}$ with no smooth isometric embedding of $\left(B_{r}, g\right)$ in $\mathbf{R}^{\mathbf{3}}$ for any $r>0$. The Gaussian curvature of $g$ changes its sign. Nadirashvili and Yuan [11] also constructed examples of non-embeddable metrics with nonpositive Gaussian curvature. Those examples clearly show that certain conditions are needed for the isometric embedding of 2-dimensional metrics in $\mathbf{R}^{\mathbf{3}}$, even locally.

The paper is organized as follows: In Section 2, we derive a differential system equivalent to the Gauss-Codazzi system. In Section 3, we study an auxiliary linear system and obtain a priori estimates for its solutions. Such a linear system is in fact related to the linearization of the Rozhdestvenskiu-Poznyak system. In Section 4, we study the linearization of our new system in a region where some directional derivative of Gaussian curvature has a fixed sign. This is the main part of the paper and the analysis is rather delicate. In Section 5, we study the Cauchy problem of the linearized system. With the assumption (A), we can put each individual region, discussed in Section 4, together to cover the whole space. In Section 6, we will use Nash-Moser-Hörmander iteration to solve our differential system and hence prove the existence of the local isometric embedding. In the last section, we shall give a brief discussion of the assumption (A) and illustrate it covers a large class of Gaussian curvature.

Acknowledgement. The project in this paper was initiated while the first two authors visited the National Center for Theoretical Sciences in Taiwan in the summer, 2000. They wish to thank NCTS for the hospitality.

\section{The fundamental equation}

The Fundamental Theorem of Surface Theory reduces an embedding of an abstract surface to the construction of a smooth solution

of the Gauss-Codazzi system. In this section, we will derive a system equivalent to the Gauss-Codazzi system. 
We denote points in $\mathbf{R}^{2}$ by $(x, t) \in \mathbf{R} \times \mathbf{R}$ and identity $\partial_{1}$ and $\partial_{2}$ with $\partial_{x}$ and $\partial_{t}$. Suppose the metric is given by

$$
I=E d x^{2}+2 F d t d x+G d t^{2},
$$

where $E, F, G$ are $C^{r}$ functions in a neighborhood of the origin. We assume that in the coordinate system $\{(x, t)\}$ the Gaussian curvature $K$ satisfies the assumption (A) in a neighborhood of the origin.

Assume that the second fundamental form of the expected isometric embedding is given by

$$
\mathrm{II}=L d x^{2}+2 M d t d x+N d t^{2} .
$$

Gauss-Codazzi system takes the following form:

$$
\begin{aligned}
& L_{t}-M_{x}=L \Gamma_{12}^{1}+M\left(\Gamma_{12}^{2}-\Gamma_{11}^{1}\right)-N \Gamma_{11}^{2}, \\
& M_{t}-N_{x}=L \Gamma_{22}^{1}+M\left(\Gamma_{22}^{2}-\Gamma_{21}^{1}\right)-N \Gamma_{21}^{2},
\end{aligned}
$$

and

$$
L N-M^{2}=K\left(E G-F^{2}\right),
$$

where $\Gamma_{i j}^{k}$ is the Christoffel symbol and $K$ is the Gaussian curvature. As usual, we set $g=\sqrt{E F-G^{2}}$.

To derive a differential system equivalent to Gauss-Codazzi system, we introduce a pair of unknown functions $(u, v)$ with $v>0$ and set

$$
L=\frac{2}{v} g, M=-\frac{u}{v} g, N=\frac{u^{2}+K v^{2}}{2 v} g .
$$

With (2.4), (2.3) is satisfied automatically. Substituting (2.4) in (2.1)$(2.2)$, we obtain

$$
\begin{aligned}
\widetilde{F}_{1}(u, v) \equiv & \partial_{t} u+\frac{1}{2} u \partial_{x} u+\frac{1}{2} K v \partial_{x} v+2 \Gamma_{22}^{1} \\
& +\left(2 \Gamma_{12}^{1}-\Gamma_{22}^{2}\right) u+\left(\frac{1}{2} \Gamma_{11}^{1}-\Gamma_{12}^{2}\right) u^{2} \\
& +\frac{1}{2}\left(\Gamma_{11}^{1} K+\partial_{x} K\right) v^{2}-\frac{1}{4} \Gamma_{11}^{2}\left(u^{2}+K v^{2}\right) u=0 \\
\widetilde{F}_{2}(u, v) \equiv & \partial_{t} v+\frac{1}{2} u \partial_{x} v-\frac{1}{2} v \partial_{x} u-\Gamma_{22}^{2} v-\Gamma_{12}^{2} u v \\
& -\frac{1}{4} \Gamma_{11}^{2}\left(u^{2}+K v^{2}\right) v=0
\end{aligned}
$$


This is based on a straightforward calculation. We omit the details and only indicate the process. By substituting (2.4) in (2.1), we get $\widetilde{F}_{2}$ first. To get $\widetilde{F}_{1}$, we substitute $(2.4)$ in $(2.2)$ and use $\widetilde{F}_{2}=0$ to replace $\partial_{t} v$.

Suppose $u$ and $v$ are solutions to (2.5) with $v>0$. Then $L, M$ and $N$ defined in (2.4) satisfy the Gauss-Codazzi system (2.1)-(2.3).

Our goal here is to solve (2.5) for $(u, v)$ with $v>0$ in a neighborhood of the origin. First we intend to find an approximate solution to the system $(2.5)$

$$
\widetilde{F}(u, v)=\left(\widetilde{F}_{1}(u, v), \widetilde{F}_{2}(u, v)\right)=0 .
$$

Since $t=0$ is not the characteristics of the system (2.5), by the Taylor expansion in $t$ we can find an approximate solution $\hat{U}=(\hat{u}, \hat{v})$ to $(2.5)$ with the initial data

$$
\hat{u}(x, 0)=0 \text { and } \hat{v}(x, 0)=1,
$$

such that $\hat{U} \in C^{r-3}$ and

$$
\widetilde{F}(\hat{u}, \hat{v})=t \widetilde{F}_{0}(x, t)
$$

for some $\widetilde{F}_{0} \in C^{r-4}$. In fact, we can choose $\hat{u}$ and $\hat{v}$ to be linear functions in $t$ in the following form:

$$
\begin{aligned}
& \hat{u}(x, t)=-\left.\left(2 \Gamma_{22}^{1}+\frac{1}{2}\left(\Gamma_{11}^{1} K+\partial_{x} K\right)\right)\right|_{(x, 0)} t, \\
& \hat{v}(x, t)=1+\left.\left(\Gamma_{22}^{2}+\frac{1}{4} \Gamma_{11}^{2} K\right)\right|_{(x, 0)} t .
\end{aligned}
$$

Introduce new variables $(x, t) \mapsto(\varepsilon x, \varepsilon t)$ and $(u, v) \mapsto(\hat{u}+\varepsilon u, \hat{v}+\varepsilon v)$. Still denote the new independent variables by $(x, t)$. The system $(2.5)$ is equivalent to

$$
F(u, v) \equiv \widetilde{F}(\hat{u}+\varepsilon u, \hat{v}+\varepsilon v)=0,
$$

with

$$
F(0,0)=\widetilde{F}(\hat{u}, \hat{v})=\varepsilon t \widetilde{F}_{0}(\varepsilon x, \varepsilon t) .
$$

By choosing $\varepsilon$ small, the system $(2.8)$ is well defined in $(-1,1) \times(-1,1)$. We may also assume that $K$ satisfies the assumption $(\mathrm{A})$ in $(-1,1) \times$ $(-1,1)$, for any $C^{1}$ function $u$ in $(-1,1) \times(-1,1)$ with small $|u|_{C^{1}}$. 
Now we extend the system (2.8) to the region

$$
\Omega=\{(x, t) ; x \in \mathbf{R},|t| \leq 1\} .
$$

Without loss of generality we may assume that all the known functions involved in (2.8) are defined in $\varepsilon \in\left(0, \varepsilon_{0}\right], t \in[-1,1], x \in \mathbf{R}$, for some small $\varepsilon_{0}$, and independent of $x$ outside a compact set of $\mathbf{R}$. We still use the same notation $\hat{u}, \hat{v}, K$, etc to denote the extended functions. For $\hat{u}$, $\hat{v}$ and $\Gamma_{i j}^{k}$, we may simply use a technique of the cutoff functions. For instance, $\hat{u}$ is replaced by $\chi \hat{u}+(1-\chi) \hat{u}(0, \cdot)$, where $\chi(x) \in C_{0}^{\infty}(\mathbf{R})$ is a cutoff function with $\chi=1$ as $|x| \leq 1 / 2$ and $\chi=0$ as $|x| \geq 1$. For the Gaussian curvature $K$, we require the following after the extension: For some positive integer $l$, some small constant $\delta>0$ and for any $C^{1}$ function $u$ in $\Omega$ with $|u|_{C^{1}}<\delta$ and $u \equiv 0$ for $|x|>1$, the region $\Omega$ is separated into finitely many subdomains by $C^{1}$ curves $\gamma_{i}: t=t_{i}(x)$, $i=1, \ldots, l$, with $t_{i}(0)=0, t_{i}^{\prime}(0) \neq t_{j}^{\prime}(0)$ for $i \neq j$ and $t_{i}(x)$ is constant for large $|x|$, and the function $\partial_{t} K+u \partial_{x} K$ changes its sign only across $\gamma_{1}, \ldots, \gamma_{l}$. Moreover, none of the curves $\gamma_{i}, i=1, \ldots, l$, is tangent to the $t$-axis or $x$-axis.

Let us compute the linearized operator of $F$ at $(\widetilde{u}, \widetilde{v})$, namely,

$$
L(u, v)=\lim _{\lambda \rightarrow 0} \frac{1}{\lambda}(F(\widetilde{u}+\lambda u, \widetilde{v}+\lambda v)-F(\widetilde{u}, \widetilde{v}))=\left(L_{1}(u, v), L_{2}(u, v)\right) .
$$

Then $L$ has the following form:

$$
L_{1}(u, v) \equiv\left(\partial_{t}+\frac{1}{2}(\hat{u}+\varepsilon \widetilde{u}) \partial_{x}\right) u+\frac{1}{2} K(\hat{v}+\varepsilon \widetilde{v}) \partial_{x} v+a_{11} u+a_{12} v,
$$

$$
L_{2}(u, v) \equiv\left(\partial_{t}+\frac{1}{2}(\hat{u}+\varepsilon \widetilde{u}) \partial_{x}\right) v-\frac{1}{2}(\hat{v}+\varepsilon \widetilde{v}) \partial_{x} u+a_{21} u+a_{22} v,
$$

where

$$
\begin{aligned}
a_{11}= & \frac{1}{2} \partial_{x} u_{*}+\varepsilon\left(2 \Gamma_{12}^{1}-\Gamma_{22}^{2}\right) \\
& +\varepsilon\left(\Gamma_{11}^{1}-2 \Gamma_{12}^{2}\right) u_{*}-\frac{1}{4} \varepsilon \Gamma_{11}^{2}\left(3 u_{*}^{2}+K v_{*}^{2}\right), \\
a_{12}= & \frac{1}{2} K \partial_{x} v_{*}+\varepsilon\left(\Gamma_{11}^{1} K+\partial_{\widetilde{x}} K\right) v_{*}-\frac{1}{2} \varepsilon \Gamma_{11}^{2} K u_{*} v_{*}, \\
a_{21}= & \frac{1}{2} \partial_{x} v_{*}-\varepsilon \Gamma_{12}^{2} v_{*}-\frac{1}{2} \varepsilon \Gamma_{11}^{2} u_{*} v_{*}, \\
a_{22}= & -\frac{1}{2} \partial_{x} u_{*}-\varepsilon \Gamma_{22}^{2}-\varepsilon \Gamma_{12}^{2} u_{*}-\frac{1}{4} \varepsilon \Gamma_{11}^{2}\left(u_{*}^{2}+3 K v_{*}^{2}\right),
\end{aligned}
$$


and

$$
u_{*}=\hat{u}+\varepsilon \widetilde{u}, \quad v_{*}=\hat{v}+\varepsilon \widetilde{v} .
$$

Here we write

$$
\partial_{\widetilde{x}} K=\left.\partial_{\widetilde{x}} K(\widetilde{x}, \widetilde{t})\right|_{(\widetilde{x}, \widetilde{t})=(\varepsilon x, \varepsilon t)} .
$$

We need to emphasize that in (2.12) all $\Gamma_{i j}^{k}$ and $K$ are evaluated at $(\varepsilon x, \varepsilon t)$.

The following result plays a crucial role in the later development:

Lemma 2.1. Suppose $K \leq 0$. Then the following hold for all $\widetilde{u}, \widetilde{v}$ with $|\widetilde{u}|_{1},|\widetilde{v}|_{1} \leq 1$ :

$$
\hat{v}+\varepsilon \widetilde{v} \geq \frac{1}{2}, \quad \text { for }(x, t) \in \mathbf{R} \times(-\mathbf{1}, \mathbf{1}), \varepsilon \in\left(\mathbf{0}, \varepsilon_{\mathbf{0}}\right],
$$

and

$$
\frac{\left|a_{12}\right|}{\sqrt{|K|}} \leq C, \quad \text { for }(x, t) \in \mathbf{R} \times(-1,1), \varepsilon \in\left(0, \varepsilon_{0}\right],
$$

where $\varepsilon_{0}$ and $C$ are two positive constants.

Proof. Since $\hat{v}(x, 0)=1$, it follows that $\hat{v}(x, t) \geq 3 / 4$ as $(x, t) \in$ $\mathbf{R} \times(-1,1)$ and $\varepsilon \in\left(0, \varepsilon_{0}\right]$ for some small positive constant $\varepsilon_{0}<1 / 4$. Hence (2.13) follows immediately for $\widetilde{v}$ with $|\widetilde{v}|_{\infty} \leq 1$. In view of the second identity in (2.12), we have

$$
\frac{\left|a_{12}\right|}{\sqrt{|K|}} \leq C\left(\sqrt{|K|}+\frac{\left|\partial_{\widetilde{x}} K\right|}{\sqrt{|K|}}\right) \leq C^{\prime},
$$

for some constant $C$ and $C^{\prime}$, since $K$ is nonpositive.

q.e.d.

Next, for each $\widetilde{u}$, introduce a transformation of coordinates

$$
T:(x, t) \longrightarrow(\xi, \eta)=(\psi(x, t), t),
$$

where $\psi$ is a solution of the following problem:

$$
\psi_{t}+\frac{1}{2}(\hat{u}+\varepsilon \widetilde{u}) \psi_{x}=0, \text { with } \psi(x, 0)=x .
$$

It is easy to see that the following result holds for $\psi$ and $T$ : 
Lemma 2.2. If $\widetilde{u} \in C^{r-3}$ in $\mathbf{R} \times(-\mathbf{1}, \mathbf{1})$, then $\psi \in C^{r-3}$ in $\mathbf{R} \times$ $(-1,1)$ and

$$
\psi_{x} \geq \frac{1}{2} \text { and }|\psi|_{r-3} \leq C\left(1+|\widetilde{u}|_{r-3}\right),
$$

for some constant $C$ independent of $\varepsilon \in\left(0, \varepsilon_{0}\right]$. Moreover, $T$ is a $C^{r-3}$ diffeomorphism in $\mathbf{R} \times[-\mathbf{1}, \mathbf{1}]$.

In the new coordinate system $\eta=t, \xi=\psi(x, t)$, we have by $(2.15)$

$$
\partial_{t}+\frac{1}{2}(\hat{u}+\varepsilon \widetilde{u}) \partial_{x}=\partial_{\eta} \text { and } \partial_{x}=\psi_{x} \partial_{\xi}
$$

With the new variables $(\xi, \eta)$, the system $(2.10)-(2.11)$ becomes

$$
\begin{aligned}
\partial_{\eta} u+\frac{1}{2} K \sigma \partial_{\xi} v+a_{11} u+a_{12} v & =g_{1}, \\
\partial_{\eta} v-\frac{1}{2} \sigma \partial_{\xi} u+a_{21} u+a_{22} v & =g_{2},
\end{aligned}
$$

where

$$
\sigma=(\hat{v}+\varepsilon \widetilde{v}) \psi_{x}
$$

is a positive function for small $\varepsilon$ by Lemma 2.1 and Lemma 2.2. The coefficients $a_{i j}, i, j=1,2$, are given in (2.12).

The system (2.18)-(2.19) is a linear hyperbolic system since $K \leq 0$ and is degenerate where $K=0$. In the next three sections, we will study the corresponding regularized system and derive a priori estimates for the Cauchy problem. By regularizing (2.18)-(2.19), we consider a new system with $K=-|K|$ replaced by $K-d=-(|K|+d)$ in (2.18)-(2.19), for small constant $d>0$. We will derive a priori estimates independent of $d$.

\section{A priori estimates on an auxiliary system}

In the following three sections, we shall derive a priori estimates for solutions associated with (2.18)-(2.19). For convenience, we shall write $(x, t)$ instead of $(\xi, \eta)$.

In this section, we shall study the following $2 \times 2$ system, defined on $(x, t) \in \mathbf{R} \times(0,1):$

$$
\begin{aligned}
& \widetilde{L}_{1}\left(w_{1}, w_{2}\right)=\partial_{\beta} w_{1}-\frac{1}{2} \partial_{\beta} \ln k\left(w_{1}-w_{2}\right)-B_{11} w_{1}-B_{12} w_{2}=g_{1} \\
& \widetilde{L}_{2}\left(w_{1}, w_{2}\right)=\partial_{\alpha} w_{2}-\frac{1}{2} \partial_{\alpha} \ln k\left(w_{2}-w_{1}\right)-B_{21} w_{1}-B_{22} w_{2}=g_{2}
\end{aligned}
$$


where $\sigma$ and $k^{2}$ are sufficiently smooth functions with $\sigma \geq \sigma_{0}$ and $0<$ $k \leq 1$ for some positive constant $\sigma_{0}$, and

$$
\partial_{\alpha}=\partial_{t}-k \sigma \partial_{x} \text { and } \partial_{\beta}=\partial_{t}+k \sigma \partial_{x} .
$$

Let $\Sigma_{ \pm}: t_{ \pm}(x) \in C^{1}[0,+\infty)$ be two curves through the origin satisfying

$$
\begin{aligned}
& 0 \leq t_{-}(x)<t_{+}(x) \leq 1 \text { for } x>0 \\
& t_{ \pm}(x)=\text { const. for large } x .
\end{aligned}
$$

Set

$$
\Omega=\left\{(x, t) ; x>0, t_{-}(x)<t<t_{+}(x)\right\},
$$

and

$$
S_{+}\left(S_{-}\right)=\left\{(x, t) \in \mathbf{R} \times(0,1) ; \partial_{t} k^{2}>0(<0)\right\} .
$$

We also write

$$
|w(x, t)|^{2}=\left|w_{1}(x, t)\right|^{2}+\left|w_{2}(x, t)\right|^{2},
$$

and sometimes denote it by $|w|^{2}$ if no confusion occurs.

In the following two results, we always assume $w$ is identically zero if $|x|$ is large.

Lemma 3.1. Let $\left(w_{1}, w_{2}\right) \in C^{1}(\bar{\Omega})$ be a solution to (3.1)-(3.2). If $t=t_{ \pm}(x)$ is space-like, i.e.,

$$
1-k \sigma\left|t_{ \pm}^{\prime}(x)\right|>0 \text { on } \Sigma_{ \pm}
$$

and $\Omega \subset \bar{S}_{-}$, then there holds for any constant $\lambda \geq 1$,

$$
\begin{aligned}
& \int_{\Sigma_{+}} e^{-\lambda t}|w|^{2}+\lambda \int_{\Omega} e^{-\lambda t}|w|^{2} \\
& \leq C\left\{\int_{\Sigma_{-}} e^{-\lambda t}|w|^{2}+\int_{\Omega} e^{-\lambda t}\left(\left|g_{1}\right|^{2}+\left|g_{2}\right|^{2}\right)\right\},
\end{aligned}
$$

where $C$ is a positive constant independent of $\lambda$ and depending only on two positive constants $C_{0}^{ \pm}$given by

$$
C_{0}^{-}=\inf _{\Sigma_{+}}\left\{\frac{1 \pm k \sigma t_{+}^{\prime}(x)}{\sqrt{1+\left[t_{+}^{\prime}(x)\right]^{2}}}\right\} \text { and } C_{0}^{+}=\sup _{\Sigma_{-}}\left\{\frac{1 \pm k \sigma t_{-}^{\prime}(x)}{\sqrt{1+\left[t_{-}^{\prime}(x)\right]^{2}}}\right\}
$$


and

$$
\sum_{i, j=1}^{2}\left|B_{i j}\right|_{\infty}+\left|\sigma \partial_{x} k\right|_{\infty}+\left|k \partial_{x} \sigma\right|_{\infty}+1
$$

Proof. Multiplying both sides of (3.1) and (3.2) by $2 e^{-\lambda t} w_{1}$ and $2 e^{-\lambda t} w_{2}$ respectively, adding them together and integrating by parts over the domain $\Omega$, we get

$$
\begin{aligned}
& \int_{\Sigma_{+}} e^{-\lambda t}\left|w_{1}\right|^{2} \frac{1+k \sigma t_{+}^{\prime}(x)}{\sqrt{1+\left[t_{+}^{\prime}(x)\right]^{2}}}+\int_{\Sigma_{+}} e^{-\lambda t}\left|w_{2}\right|^{2} \frac{1-k \sigma t_{+}^{\prime}(x)}{\sqrt{1+\left[t_{+}^{\prime}(x)\right]^{2}}} \\
& +\lambda \int_{\Omega} e^{-\lambda t}|w|^{2} d x d t-\int_{\Omega} e^{-\lambda t} \partial_{t} \ln k\left(w_{1}-w_{2}\right)^{2} \\
& =\int_{\Sigma_{-}} e^{-\lambda t}\left|w_{1}\right|^{2} \frac{1+k \sigma t_{-}^{\prime}(x)}{\sqrt{1+\left[t_{-}^{\prime}(x)\right]^{2}}}+\int_{\Sigma_{-}} e^{-\lambda t}\left|w_{2}\right|^{2} \frac{1-k \sigma t_{-}^{\prime}(x)}{\sqrt{1+\left[t_{-}^{\prime}(x)\right]^{2}}} \\
& \quad+2 \int_{\Omega} e^{-\lambda t}\left\{\left(\partial_{x} k \sigma+\frac{1}{2} k \partial_{x} \sigma\right)\left(w_{1}^{2}-w_{2}^{2}\right)+B_{11} w_{1}^{2}\right. \\
& \left.\quad+\left(B_{12}+B_{21}\right) w_{1} w_{2}+B_{22} w_{2}^{2}\right\}+2 \int_{\Omega} e^{-\lambda t}\left(g_{1} w_{1}+g_{2} w_{2}\right) .
\end{aligned}
$$

In view of the hypothesis $\Omega \subset \bar{S}_{-}$, the fourth term in the left-hand side of (3.11) is nonnegative. Moreover, the third term in the right-hand side of (3.11) is controlled by

$$
C \int_{\Omega} e^{-\lambda t}|w|^{2}
$$

for some constant $C$ depending only on the quantities in (3.10). By noting the definition of $C_{0}^{ \pm}$in (3.9), we obtain

$$
\begin{aligned}
& C_{0}^{-} \int_{\Sigma_{+}} e^{-\lambda t}|w|^{2}+(\lambda-C) \int_{\Omega} e^{-\lambda t}|w|^{2} \\
& \leq C_{0}^{+} \int_{\Sigma_{-}} e^{-\lambda t}|w|^{2}+\int_{\Omega} e^{-\lambda t}\left(\left|g_{1}\right|^{2}+\left|g_{2}\right|^{2}\right),
\end{aligned}
$$

where $C$ is a positive constant independent of $\lambda$ and depending only on (3.10). Thus (3.8) is proved if we rename $\lambda$. 
Lemma 3.2. Suppose that $\Omega \subset \bar{S}_{+}$and all the remaining assumptions in Lemma 3.1 are fulfilled. Then the following holds for any constant $\lambda \geq 1$ :

$$
\begin{aligned}
& \int_{\Sigma_{+}} e^{-\lambda t}\left|\frac{w}{k}\right|^{2}+\lambda \int_{\Omega} e^{-\lambda t}\left|\frac{w}{k}\right|^{2} \\
& \leq C\left\{\int_{\Sigma_{-}} e^{-\lambda t}\left|\frac{w}{k}\right|^{2}+\int_{\Omega} e^{-\lambda t}\left|\frac{g}{k}\right|^{2}\right\},
\end{aligned}
$$

where $C$ is a positive constant depending only on the quantities in (3.9) and (3.10).

Proof. From (3.1)-(3.2), $\widetilde{w}_{i}=w_{i} / k, i=1,2$, satisfies the following system:

$$
\begin{aligned}
& \partial_{\beta} \widetilde{w}_{1}+\frac{1}{2} \partial_{\beta} \ln k\left(\widetilde{w}_{1}+\widetilde{w}_{2}\right)-B_{11} \widetilde{w}_{1}-B_{12} \widetilde{w}_{2}=\frac{1}{k} g_{1}, \\
& \partial_{\alpha} \widetilde{w}_{2}+\frac{1}{2} \partial_{\alpha} \ln k\left(\widetilde{w}_{2}+\widetilde{w}_{1}\right)-B_{21} \widetilde{w}_{1}-B_{22} \widetilde{w}_{2}=\frac{1}{k} g_{2} .
\end{aligned}
$$

Multiplying both sides of (3.13) and (3.14) by $2 e^{-\lambda t} \widetilde{w}_{1}$ and $2 e^{-\lambda t} \widetilde{w}_{2}$ respectively. Then proceed as in the proof of Lemma 3.1 to get (3.12). We omit the details.

\section{A priori estimates on the linearized system}

In this section, we will study the following linear system, defined in $(x, t) \in \mathbf{R} \times(0,1):$

$$
\begin{aligned}
& L_{1}(u, v)=\partial_{t} u-k^{2} \sigma \partial_{x} v+a_{11} u+a_{12} v=g_{1}, \\
& L_{2}(u, v)=\partial_{t} v-\sigma \partial_{x} u+a_{21} u+a_{22} v=g_{2},
\end{aligned}
$$

with

$$
\left.u\right|_{\Sigma_{-}}=u_{0},\left.v\right|_{\Sigma_{-}}=v_{0} .
$$

Here $k$ and $\sigma$ are assumed as in Section 3. And we also use the notations of (3.4), (3.5) and (3.6). Our goal in this section is to derive estimates for solutions in $\Omega$ independent of $\inf k$. Again, we assume solutions $(u, v)$ are zero for large $|x|$.

The characteristic equation of (4.1)-(4.2) is given by

$$
\operatorname{det}\left(\begin{array}{cc}
\lambda & \sigma k^{2} \\
\sigma & \lambda
\end{array}\right)=\lambda^{2}-k^{2} \sigma^{2}=0
$$


Hence its eigenvalues are $\lambda_{ \pm}= \pm k \sigma$ and the corresponding left eigenvectors are $(1,-k)^{t}$ and $(1, k)^{t}$. Denote its Riemann invariant by $w_{1}=u-k v$ and $w_{2}=u+k v$. Computing $L_{1} \mp k L_{2}$ we derive that $w_{1}$ and $w_{2}$ satisfy a $2 \times 2$ system

$$
\begin{aligned}
& \widetilde{L}_{1}\left(w_{1}, w_{2}\right)=\partial_{\beta} w_{1}-\frac{1}{2} \partial_{\beta} \ln k\left(w_{1}-w_{2}\right)-B_{11} w_{1}-B_{12} w_{2}=\widetilde{g}_{1} \\
& \widetilde{L}_{2}\left(w_{1}, w_{2}\right)=\partial_{\alpha} w_{2}-\frac{1}{2} \partial_{\alpha} \ln k\left(w_{2}-w_{1}\right)-B_{21} w_{1}-B_{22} w_{2}=\widetilde{g}_{2}
\end{aligned}
$$

where

$$
\widetilde{g}_{1}=g_{1}-k g_{2}, \widetilde{g}_{2}=g_{1}+k g_{2},
$$

and

$$
\begin{aligned}
& B_{11}=\frac{1}{2}\left(-a_{11}+\frac{a_{12}}{k}+k a_{21}-a_{22}\right), \\
& B_{12}=\frac{1}{2}\left(-a_{11}-\frac{a_{12}}{k}+k a_{21}+a_{22}\right), \\
& B_{21}=\frac{1}{2}\left(-a_{11}+\frac{a_{12}}{k}-k a_{21}+a_{22}\right), \\
& B_{22}=\frac{1}{2}\left(-a_{11}-\frac{a_{12}}{k}-k a_{21}-a_{22}\right) .
\end{aligned}
$$

For $U=(u, v)$, set

$$
|U|^{2}=|u|^{2}+|v|^{2},
$$

and for $s \geq 1$

$$
\begin{aligned}
& |U|_{s}^{2}=\sum_{|\gamma| \leq s}\left(\left|\partial^{\gamma} u\right|^{2}+\left|\partial^{\gamma} v\right|^{2}\right), \\
& |U|_{(0, s)}^{2}=\sum_{j=0}^{s}\left(\left|\partial_{x}^{j} u\right|^{2}+\left|\partial_{x}^{j} v\right|^{2}\right) .
\end{aligned}
$$

Now we derive an estimate of solutions to (4.1)-(4.2) in the region where $\partial_{t} k \geq 0$. We compare our solutions with the solution to a single differential equation. The difference satisfies the system studied in the previous section. 
Lemma 4.1. Let $(u, v)$ be a $C^{1}(\bar{\Omega})$ solution to (4.1)-(4.2) with $a_{i j}$, $\sigma, k^{2} \in C^{1}(\bar{\Omega})$ and $k \in \operatorname{Lip}(\Omega)$, with $0<k \leq 1$ and $\sigma \geq \sigma_{0}$ for some positive constant $\sigma_{0}$. Suppose $\Omega \subset \bar{S}_{+}$and (3.7) is fulfilled. Then for any $\lambda \geq 1$

$$
\begin{aligned}
& \int_{\Sigma_{+}} e^{-\lambda t}|U|^{2}+\lambda \int_{\Omega} e^{-\lambda t}|U|^{2} \\
& \leq C\left\{\int_{\Sigma_{-}} e^{-\lambda t}|U|_{1}^{2}+\int_{\Omega} e^{-\lambda t}\left(|G|^{2}+\left|\partial_{x} G\right|^{2}\right)\right\},
\end{aligned}
$$

where $C$ is a positive constant depending only on

$$
\theta \equiv\left|t_{ \pm}^{\prime}(x)\right|_{\infty}+\left|\partial_{x} k\right|_{\infty}+\sup _{\Omega} \frac{\left|a_{12}\right|}{k}<\infty
$$

and

$$
\sum_{i, j=1}^{2} \sum_{n=0}^{1}\left|\partial_{x}^{n} a_{i j}\right|_{\infty}+\sum_{n=0}^{1}\left(\left|\partial_{x}^{n} \sigma\right|_{\infty}+\left|\partial_{x}^{n} k^{2}\right|_{\infty}\right) .
$$

Proof. Consider the Cauchy problem

$$
\begin{aligned}
\partial_{\beta} z-\left(B_{11}+B_{12}\right) z & =g_{1} \text { in } \Omega, \\
z & =u_{0} \text { on } \Sigma_{-} .
\end{aligned}
$$

Note $B_{11}+B_{12}=k a_{21}-a_{11}$. First we have

$$
\int_{\Sigma_{+}} e^{-\lambda t}|z|^{2}+\lambda \int_{\Omega} e^{-\lambda t}|z|^{2} \leq C\left\{\int_{\Sigma_{-}} e^{-\lambda t}|u|^{2}+\int_{\Omega} e^{-\lambda t}\left|g_{1}\right|^{2}\right\},
$$

for some constant $C$ depending only on the quantities in (4.7) and (4.8). We obtain (4.10) by multiplying $2 e^{-\lambda t} z$ to (4.9) and integrating over $\Omega$ as in the proof of Lemma 3.1. We omit the detail since it is similar and only easier. Now we differentiate (4.9) with respect to $x$ and consider the equation satisfied by $\partial_{x} z$. Similarly we get

$$
\int_{\Omega} e^{-\lambda t}\left|\partial_{x} z\right|^{2} \leq C\left\{\int_{\Sigma_{-}} e^{-\lambda t}|U|_{1}^{2}+\int_{\Omega} e^{-\lambda t}\left(\left|g_{1}\right|^{2}+\left|\partial_{x} g_{1}\right|^{2}\right)\right\}
$$

We remark that by (4.1) and (4.9) $\partial z$ on $\Sigma_{-}$can be expressed by a linear combination of $u, v$ and their derivatives restricted on $\Sigma_{-}$. 
Introducing unknown functions $\widetilde{w}_{i}+z=w_{i}$, by (3.3), (4.5), (4.6) and (4.9) we obtain

$$
\begin{aligned}
& \widetilde{L}_{1}\left(\widetilde{w}_{1}, \widetilde{w}_{2}\right)=-k g_{2}, \\
& \widetilde{L}_{2}\left(\widetilde{w}_{1}, \widetilde{w}_{2}\right)=k\left(g_{2}+2 \sigma \partial_{x} z-2 a_{21} z\right) .
\end{aligned}
$$

Moreover, $\widetilde{w}_{i}, i=1,2$, is subject to

$$
\widetilde{w}_{1}=-k v_{0} \text { and } \widetilde{w}_{2}=k v_{0} \text { on } \Sigma_{-} .
$$

An application of Lemma 3.2 to (4.12)-(4.13) in $\Omega$ yields

$$
\begin{aligned}
& \int_{\Sigma_{+}} e^{-\lambda t}\left|\frac{\widetilde{w}}{k}\right|^{2}+\lambda \int_{\Omega} e^{-\lambda t}\left|\frac{\widetilde{w}}{k}\right|^{2} \\
& \leq C\left\{\int_{\Sigma_{-}} e^{-\lambda t}|v|^{2}+\int_{\Omega} e^{-\lambda t}\left(\left|g_{2}\right|^{2}+|z|^{2}+\left|\partial_{x} z\right|^{2}\right)\right\} .
\end{aligned}
$$

With the definition $\widetilde{w}_{i}+z=w_{i}=u \pm k v$, we get

$$
\begin{aligned}
& \int_{\Sigma_{+}} e^{-\lambda t}\left(|u|^{2}+|v|^{2}\right)+\lambda \int_{\Omega} e^{-\lambda t}\left(|u|^{2}+|v|^{2}\right) \\
& \leq C\left\{\int_{\Sigma_{-}} e^{-\lambda t}|v|^{2}+\int_{\Sigma_{+}} e^{-\lambda t}|z|^{2}+\int_{\Omega} e^{-\lambda t}\left(\left|g_{2}\right|^{2}+\lambda|z|^{2}+\left|\partial_{x} z\right|^{2}\right)\right\} .
\end{aligned}
$$

We finish the proof by substituting (4.10) and (4.11) in (4.14). q.e.d.

Now we study the case when $\partial_{t} k \leq 0$. For our solution $(u, v)$, the $u$ component is good and there is a degeneracy in the $v$ component. In order to get an estimate on $v$, we need information of $u$ and its $x$ derivative.

Lemma 4.2. Let $(u, v)$ be a $C^{1}(\bar{\Omega})$ solution to (4.1)-(4.2) with $a_{i j}$, $\sigma, k^{2} \in C^{1}(\bar{\Omega})$ and $k \in \operatorname{Lip}(\Omega)$, with $0<k \leq 1$ and $\sigma \geq \sigma_{0}$ for some positive constant $\sigma_{0}$. Suppose $\Omega \subset \bar{S}_{-}$and (3.7) is fulfilled. Then for any $\lambda \geq 1$,

$$
\begin{aligned}
& \int_{\Sigma_{+}} e^{-\lambda t}|U|^{2}+\lambda \int_{\Omega} e^{-\lambda t}|U|^{2} \\
& \leq C\left\{\int_{\Sigma_{-}} e^{-\lambda t}|U|_{1}^{2}+\int_{\Omega} e^{-\lambda t}\left(|G|^{2}+\left|\partial_{x} G\right|^{2}\right)\right\},
\end{aligned}
$$

where $C$ is a positive constant depending only on the quantities in (4.7) and (4.8). 
Proof. From (4.3)-(4.4) and Lemma 3.1, it follows that

$$
\begin{aligned}
& \int_{\Sigma_{+}} e^{-\lambda t}\left(|u|^{2}+|k v|^{2}\right)+\lambda \int_{\Omega} e^{-\lambda t}\left(|u|^{2}+|k v|^{2}\right) \\
& \leq C\left\{\int_{\Sigma_{-}} e^{-\lambda t}\left(|u|^{2}+|k v|^{2}\right)+\int_{\Omega} e^{-\lambda t}\left(\left|g_{1}\right|^{2}+\left|g_{2}\right|^{2}\right)\right\} .
\end{aligned}
$$

In order to get an estimate without the weight $k$, we have to return to the original system (4.1)-(4.2). Differentiation of (4.1)-(4.2) in $x$ yields the system satisfied by $u_{1}=u_{x}$ and $v_{1}=v_{x}$

$\partial_{t} u_{1}-k^{2} \sigma \partial_{x} v_{1}+a_{11} u_{1}+\left(a_{12}-\left(k^{2} \sigma\right)_{x}\right) v_{1}=\partial_{x} g_{1}-\partial_{x} a_{11} u-\partial_{x} a_{12} v$,

$$
\partial_{t} v_{1}-\sigma \partial_{x} u_{1}+\left(a_{21}-\partial_{x} \sigma\right) u_{1}+a_{22} v_{1}=\partial_{x} g_{2}-\partial_{x} a_{21} u-\partial_{x} a_{22} v .
$$

The system (4.16)-(4.17) has the same structure as (4.1)-(4.2). A similar result as $(4.15)$ holds for $\left(u_{1}, v_{1}\right)$. Hence, we have

$$
\begin{aligned}
& \int_{\Sigma_{+}} e^{-\lambda t}\left(\left|u_{1}\right|^{2}+\left|k v_{1}\right|^{2}\right)+\lambda \int_{\Omega} e^{-\lambda t}\left(\left|u_{1}\right|^{2}+\left|k v_{1}\right|^{2}\right) \\
& \leq C\left\{\int_{\Sigma_{-}} e^{-\lambda t}\left(\left|u_{1}\right|^{2}+\left|k v_{1}\right|^{2}\right)+\int_{\Omega} e^{-\lambda t}\left(\left|\partial_{x} g_{1}\right|^{2}+\left|\partial_{x} g_{2}\right|^{2}\right)\right\} \\
& \quad+C \int_{\Omega} e^{-\lambda t}\left(|u|^{2}+|v|^{2}\right) .
\end{aligned}
$$

We remark that $\left.u_{1}\right|_{\Sigma_{-}}$and $\left.v_{1}\right|_{\Sigma_{-}}$can be estimated in terms of $u_{0}, v_{0}$ and their tangential derivatives along $\Sigma_{-}$, with the help of (4.1)-(4.2).

Multiplying (4.2) by $2 e^{-\lambda t} v$, we get

$$
\left(e^{-\lambda t} v^{2}\right)_{t}+\left(\lambda+2 a_{22}\right) e^{-\lambda t} v^{2}=2 e^{-\lambda t} g_{2} v+2 \sigma e^{-\lambda t} u_{1} v-2 a_{21} e^{-\lambda t} u v
$$

Integrating (4.19) over $\Omega$ and using the Cauchy inequality, we get

$$
\begin{aligned}
& \int_{\Sigma_{+}} e^{-\lambda t} v^{2}+\lambda \int_{\Omega} e^{-\lambda t} v^{2} \\
& \leq \int_{\Sigma_{-}} e^{-\lambda t} v^{2}+C \int_{\Omega} e^{-\lambda t}\left(\left|u_{1}\right|^{2}+|u|^{2}+\left|g_{2}\right|^{2}\right),
\end{aligned}
$$


for any $\lambda \geq 1$, after renaming it if necessary, and some constant $C$ depending only the quantities defined in (4.8). Summation of (4.15), (4.18) and (4.20) yields

$$
\begin{aligned}
& \int_{\Sigma_{+}} e^{-\lambda t}|U|^{2}+\lambda \int_{\Omega} e^{-\lambda t}|U|^{2} \\
& \leq C\left\{\int_{\Sigma_{-}} e^{-\lambda t}|U|_{1}^{2}+\int_{\Omega} e^{-\lambda t}\left(|G|^{2}+\left|\partial_{x} G\right|^{2}\right)\right\} .
\end{aligned}
$$

This finishes the proof.

q.e.d.

Remark. The results in Lemma 4.1 and Lemma 4.2 have similar forms. However, they are established by totally different methods. Lemma 4.1 is proved by a straightforward application of Lemma 3.2. For Lemma 4.2, we need to apply Lemma 3.1 to the system satisfied by $(u, v)$ and the system satisfied by $x$-derivative of $(u, v)$ in order to get the estimate on $(u, v)$ itself.

In the rest of this section we intend to establish the estimates for higher order derivatives of solutions to the system (4.1)-(4.2). With $u_{s}=\partial_{x}^{s} u$ and $v_{s}=\partial_{x}^{s} v, s$-times differentiation of (4.1) and (4.2) yields

$$
\begin{aligned}
& L_{1}\left(u_{s}, v_{s}\right)=\partial_{t} u_{s}-k^{2} \sigma \partial_{x} v_{s}+a_{11} u_{s}+\widetilde{a}_{12} v_{s}=g_{1 s}+\partial_{x}^{s} g_{1}, \\
& L_{2}\left(u_{s}, v_{s}\right)=\partial_{t} v_{s}-\sigma \partial_{x} u_{s}+\widetilde{a}_{21} u_{s}+a_{22} v_{s}=g_{2 s}+\partial_{x}^{s} g_{2},
\end{aligned}
$$

where

$$
\widetilde{a}_{12}=a_{12}-s \partial_{x}\left(k^{2} \sigma\right), \quad \widetilde{a}_{21}=a_{21}-s \partial_{x} \sigma,
$$

and

$$
\begin{aligned}
& g_{1 s}=\sum_{i+i^{\prime}=s, i \geq 1} C_{i}\left\{(i-1) \partial_{x}^{i}\left(k^{2} \sigma\right) \partial_{x}^{i^{\prime}+1} v-\left(\partial_{x}^{i} a_{11} \partial_{x}^{i^{\prime}} u+\partial_{x}^{i} a_{12} \partial_{x}^{i^{\prime}} v\right)\right\} \\
& g_{2 s}=\sum_{i+i^{\prime}=s, i \geq 1} C_{i}\left\{(i-1) \partial_{x}^{i} \sigma \partial_{x}^{i^{\prime}+1} u-\left(\partial_{x}^{i} a_{21} \partial_{x}^{i^{\prime}} u+\partial_{x}^{i} a_{22} \partial_{x}^{i^{\prime}} v\right)\right\} .
\end{aligned}
$$

Notice that

$$
\frac{\left|\widetilde{a}_{12}\right|}{k} \leq \frac{\left|a_{12}\right|}{k}+C_{1}
$$

for some constant $C_{1}$ depending only on $\theta$. Hence the requirements on $\widetilde{a}_{12}$ in Lemma 4.1 and Lemma 4.2 are fulfilled. We also note that in (4.23) and (4.24) the highest derivatives of $u$ and $v$ are $\partial_{x}^{s-1} u$ and $\partial_{x}^{s-1} u$. This fact will be used in (4.28). 
Lemma 4.3. Let $s \geq 1$ be an integer and $(u, v)$ be a $C^{s+1}(\bar{\Omega})$ solution to (4.1)-(4.2) with $a_{i j}, \sigma, k^{2} \in C^{s+1}(\bar{\Omega})$ and $k \in \operatorname{Lip}(\Omega)$, with $0<k \leq 1$ and $\sigma \geq \sigma_{0}$ for some positive constant $\sigma_{0}$. Suppose $\Omega \subset \bar{S}_{+}$ (or $\Omega \subset \bar{S}_{-}$) and (3.7) is fulfilled. Then for each $\lambda \geq 1$,

$$
\begin{aligned}
& \int_{\Sigma_{+}} e^{-\lambda t}|U|_{s}^{2}+\lambda \int_{\Omega} e^{-\lambda t}|U|_{s}^{2} \\
& \leq C_{s}\left\{\int_{\Sigma_{-}} e^{-\lambda t}|U|_{s+1}^{2}+\int_{\Sigma_{+}} e^{-\lambda t}|G|_{s-1}^{2}\right. \\
& \left.\quad+\int_{\Omega} e^{-\lambda t}\left(|G|_{(0, s+1)}^{2}+\lambda|G|_{s-1}^{2}\right)\right\},
\end{aligned}
$$

where $C_{s}$ is a positive constant depending only on $\theta$ in (4.7) and

$$
\sum_{i, j=1}^{2} \sum_{|\gamma|=0}^{s+1}\left|\partial^{\gamma} a_{i j}\right|_{\infty}+\sum_{|\gamma|=0}^{s+1}\left(\left|\partial^{\gamma} \sigma\right|_{\infty}+\left|\partial^{\gamma} k^{2}\right|_{\infty}\right) .
$$

Proof. We shall prove (4.25) by induction. (4.25) 0 is just Lemma 4.1 (or Lemma 4.2). Assume $(4.25)_{j}$ is true for all $j \leq s-1$ and $s \geq 1$. An application of Lemma 4.1 (or Lemma 4.2) to (4.21)-(4.22) in $\Omega$ yields

$$
\begin{aligned}
& \int_{\Sigma_{+}} e^{-\lambda t}\left|U_{s}\right|^{2}+\lambda \int_{\Omega} e^{-\lambda t}\left|U_{s}\right|^{2} \\
& \leq C\left\{\int_{\Sigma_{-}} e^{-\lambda t}\left|U_{s}\right|_{1}^{2}+\int_{\Omega} e^{-\lambda t}\left(\left|G_{s}\right|^{2}+\left|G_{s+1}\right|^{2}\right)\right\} \\
& \quad+C\left\{\int_{\Omega} e^{-\lambda t}\left(\left|g_{1 s}\right|^{2}+\left|g_{2 s}\right|^{2}+\left|\partial_{x} g_{1 s}\right|^{2}+\left|\partial_{x} g_{2 s}\right|^{2}\right)\right\} \\
& =I_{1}+I_{2}
\end{aligned}
$$

for some constant $C$ depending only on the quantities (4.7) and (4.8). It suffices to evaluate $I_{2}$. From (4.23) and (4.24), it is easy to see

$$
I_{2} \leq C \int_{\Omega} e^{-\lambda t} \sum_{j=0}^{s}\left|U_{j}\right|^{2}
$$

for some constant $C$ depending only on the quantities in (4.26). Combining (4.27) and (4.28), we have

$$
\begin{aligned}
& \int_{\Sigma_{+}} e^{-\lambda t}\left|U_{s}\right|^{2}+\lambda \int_{\Omega} e^{-\lambda t}\left|U_{s}\right|^{2} \\
& \leq C\left\{\int_{\Sigma_{-}} e^{-\lambda t}\left|U_{s}\right|_{1}^{2}+\int_{\Omega} e^{-\lambda t}|G|_{(0, s+1)}^{2}\right\}+C \int_{\Omega} e^{-\lambda t}|U|_{(0, s)}^{2},
\end{aligned}
$$


for some constant $C$ depending only on the quantities in (4.26) and $\theta$ in (4.7). By renaming $\lambda$, we obtain

$$
\begin{aligned}
& \int_{\Sigma_{+}} e^{-\lambda t}\left|U_{s}\right|^{2}+\lambda \int_{\Omega} e^{-\lambda t}\left|U_{s}\right|^{2} \\
& \leq C\left\{\int_{\Sigma_{-}} e^{-\lambda t}\left|U_{s}\right|_{1}^{2}+\int_{\Omega} e^{-\lambda t}|G|_{(0, s+1)}^{2}\right\}+C \int_{\Omega} e^{-\lambda t}|U|_{(0, s-1)}^{2} .
\end{aligned}
$$

It remains to evaluate the $L^{2}$-integral of $\partial_{t}^{j} \partial_{x}^{s-j} U$ for $1 \leq j \leq s$. From (4.1)-(4.2) it follows that

$$
\begin{aligned}
& \partial_{t} \partial_{x}^{s-1} u=\partial_{x}^{s-1}\left(k^{2} \sigma \partial_{x} v\right)-\partial_{x}^{s-1}\left(a_{11} u\right)-\partial_{x}^{s-1}\left(a_{12} v\right)+\partial_{x}^{s-1} g_{1}, \\
& \partial_{t} \partial_{x}^{s-1} v=\partial_{x}^{s-1}\left(\sigma \partial_{x} u\right)-\partial_{x}^{s-1}\left(a_{21} u\right)-\partial_{x}^{s-1}\left(a_{12} v\right)+\partial_{x}^{s-1} g_{2} .
\end{aligned}
$$

Hence, we get

$$
\left|\partial_{t} U_{s-1}\right|^{2} \leq C\left(|U|_{(0, s)}^{2}+\left|G_{s-1}\right|^{2}\right)
$$

for some constant $C$ depending only on the quantities in (4.26). And similarly, by induction we obtain for $j=1, \ldots, s$

$$
\left|\partial_{t}^{j} U_{s-j}\right|^{2} \leq C\left(|U|_{(0, s)}^{2}+|G|_{s-1}^{2}\right) .
$$

This implies

$$
\begin{aligned}
& \sum_{j=1}^{s} \int_{\Sigma_{+}} e^{-\lambda t}\left|\partial_{t}^{j} U_{s-j}\right|^{2}+\lambda \sum_{j=1}^{s} \int_{\Omega} e^{-\lambda t}\left|\partial_{t}^{j} U_{s-j}\right|^{2} \\
& \leq C\left\{\int_{\Sigma_{+}} e^{-\lambda t}|G|_{s-1}^{2}+\lambda \int_{\Omega} e^{-\lambda t}|G|_{s-1}^{2}\right\} \\
& \quad+C\left\{\int_{\Sigma_{+}} e^{-\lambda t}|U|_{(0, s)}^{2}+\lambda \int_{\Omega} e^{-\lambda t}|U|_{(0, s)}^{2}\right\} .
\end{aligned}
$$

Combining (4.29) and (4.30), we get

$$
\begin{aligned}
& \sum_{j=0}^{s} \int_{\Sigma_{+}} e^{-\lambda t}\left|\partial_{t}^{j} U_{s-j}\right|^{2}+\lambda \sum_{j=0}^{s} \int_{\Omega} e^{-\lambda t}\left|\partial_{t}^{j} U_{s-j}\right|^{2} \\
& \leq C^{\prime}\left\{\int_{\Sigma_{-}} e^{-\lambda t}|U|_{s+1}^{2}+\int_{\Sigma_{+}} e^{-\lambda t}|G|_{s-1}^{2}+\int_{\Omega} e^{-\lambda t}\left(|G|_{(0, s+1)}^{2}+\lambda|G|_{s-1}^{2}\right)\right\} \\
& \quad+C^{\prime}\left\{\int_{\Sigma_{+}} e^{-\lambda t}|U|_{(0, s-1)}^{2}+\lambda \int_{\Omega} e^{-\lambda t}|U|_{(0, s-1)}^{2}\right\}
\end{aligned}
$$


Notice that

$$
|U|_{s}^{2}=\sum_{j=0}^{s}\left|\partial_{t}^{j} \partial_{x}^{s-j} U\right|^{2}+\sum_{j=0}^{s-1}|U|_{j}^{2} .
$$

It follows

$$
\begin{aligned}
& \int_{\Sigma_{+}} e^{-\lambda t}|U|_{s}^{2}+\lambda \int_{\Omega} e^{-\lambda t}|U|_{s}^{2} \\
& \leq C\left\{\int_{\Sigma_{-}} e^{-\lambda t}|U|_{s+1}^{2}+\int_{\Sigma_{+}} e^{-\lambda t}|G|_{s-1}^{2}+\int_{\Omega} e^{-\lambda t}\left(|G|_{(0, s+1)}^{2}+\lambda|G|_{s-1}^{2}\right)\right\} \\
& +C\left\{\int_{\Sigma_{+}} e^{-\lambda t}|U|_{s-1}^{2}+\lambda \int_{\Omega} e^{-\lambda t}|U|_{s-1}^{2}\right\} .
\end{aligned}
$$

We finish the proof by the hypothesis on induction.

q.e.d.

Remark. If $\Sigma_{-}=\{t=0\}$, then in (4.25) the term

$$
\int_{t=0}|U|_{s+1}^{2}
$$

can be replaced by

$$
\int_{t=0}|U|_{(0, s+1)}^{2}
$$

This follows by checking the proof of Lemma 4.1, 4.2 and 4.3. This fact will be used in the next section.

\section{A priori estimates for the Cauchy problem}

In this section, we proceed to study a Cauchy problem, defined in $(x, t) \in \mathbf{R} \times(-1,1)$,

$$
\begin{aligned}
& L_{1}(u, v)=\partial_{t} u-k^{2} \sigma \partial_{x} v+a_{11} u+a_{12} v=g_{1}, \\
& L_{2}(u, v)=\partial_{t} v-\sigma \partial_{x} u+a_{21} u+a_{22} v=g_{2},
\end{aligned}
$$

with

$$
u(x, 0)=u_{0}(x), v(x, 0)=v_{0}(x) .
$$

We shall derive estimates of solutions independent of inf $k$. Again, we assume all solutions have compact supports in $x$. We set

$$
\Omega=\{(x, t) ; x \in \mathbf{R}, t \in(-1,1)\} .
$$


In the following, we always assume $\varepsilon$ is so small that Lemma 2.1 and 2.2 hold. For convenience, we still use $(x, t)$ instead of $(\xi, \eta)$.

For any function $\widetilde{U}=(\widetilde{u}, \widetilde{v})$, we assume $a_{i j}$ is given in terms of $\widetilde{U}$ in (2.12), with

$$
K=-k^{2},
$$

and $\sigma$ is given in (2.20). It is easy to see for $s=m$ the quantities in (4.26) is bounded if $|\widetilde{U}|_{m+2} \leq 1$.

We first consider $t \geq 0$ and set

$$
\Omega^{+}=\Omega \cap\{t \geq 0\} .
$$

Suppose that there are $l_{+}+l_{-}$curves: $t_{i}^{-} \in C^{1}(-\infty, 0], i=1, \ldots, l_{-}$, and $t_{j}^{+} \in C^{1}[0, \infty), j=1, \ldots, l_{+}$, subject to $t_{i}^{-}(0)=0$ and $t_{j}^{+}(0)=0$, equal to constants outside a compact set of $\mathbf{R}$ and

$$
\begin{aligned}
& 0<t_{1}^{+}(x)<t_{2}^{+}(x)<\cdots<t_{l_{+}}^{+}(x) \text { as } x>0, \\
& 0<t_{1}^{-}(x)<t_{2}^{-}(x)<\cdots<t_{l_{-}}^{-}(x) \text { as } x<0 .
\end{aligned}
$$

We assume for some positive constants $c_{1}$ and $c_{2}$

$$
c_{1} \leq \inf _{t=t_{j}^{ \pm}(x)}\left\{\frac{1-\left|k \sigma\left(t_{j}^{ \pm}\right)^{\prime}(x)\right|}{\sqrt{1+\left[\left(t_{j}^{ \pm}\right)^{\prime}(x)\right]^{2}}}\right\} \leq \sup _{t=t_{j}^{ \pm}(x)}\left\{\frac{1+\left|k \sigma\left(t_{j}^{ \pm}\right)^{\prime}(x)\right|}{\sqrt{1+\left[\left(t_{j}^{ \pm}\right)^{\prime}(x)\right]^{2}}}\right\} \leq c_{2},
$$

for any $j=1, \ldots, l_{ \pm}$. By denoting

$$
t_{0}^{ \pm}(x)=0 \text { and } t_{l_{ \pm}+1}^{ \pm}(x)=1,
$$

we set for $0 \leq j \leq \min \left\{l_{+}, l_{-}\right\}$,

$$
\Omega_{j}^{+}=\left\{(x, t) \in \Omega^{+} ; \quad \begin{array}{l}
t_{l_{+}-j}^{+}(x)<t<t_{l_{+}-j+1}^{+}(x), x \geq 0 \\
t_{l_{-}-j}(x)<t<t_{l_{-}-j+1}(x), x \leq 0
\end{array}\right\}
$$

and

$$
\Sigma_{j}^{+}=\left\{(x, t) \in \Omega^{+} ; t=t_{l_{+}-j}^{+}(x), x \geq 0 \text { and } t=t_{l_{-}-j}^{-}(x), x<0\right\} .
$$

For the case $l_{-} \neq l_{+}$, we assume $l_{+}>l_{-}$without loss of generality. Then we set for $l_{-}<j \leq l_{+}$

$$
\Omega_{j}^{+}=\left\{(x, t) \in \Omega^{+} ; t_{l_{+}-j}^{+}(x)<t<t_{l_{+}-j+1}^{+}(x), x \geq 0\right\},
$$


and

$$
\Sigma_{j}^{+}=\left\{(x, t) \in \Omega^{+} ; t=t_{l_{+}-j}^{+}(x), x \geq 0\right\} .
$$

The case $l_{+}<l_{-}$can be discussed similarly. Therefore, with $m^{+}=$ $\max \left\{l_{+}, l_{-}\right\}$, we have the decompostion

$$
\Omega^{+}=\bigcup_{j=1}^{m^{+}} \Omega_{j}^{+} .
$$

For $t \leq 0$, we assume we can decompose $\Omega^{-}=\Omega \cap\{t \leq 0\}$ in a similar way

$$
\Omega^{-}=\bigcup_{j=1}^{m^{-}} \Omega_{j}^{-} .
$$

We assume the following alternative for $j=1, \ldots, m^{ \pm}$:

$$
\Omega_{j}^{ \pm} \subset \bar{S}_{+} \text {or } \Omega_{j}^{ \pm} \subset \bar{S}_{-}
$$

where

$$
S_{+}\left(S_{-}\right)=\left\{(x, t) \in \Omega ; \partial_{t} k^{2}>0(<0)\right\}
$$

Define for $\lambda>0$

$$
\begin{aligned}
\|\mid U\|_{\lambda, s}^{2} & =\sum_{j=0}^{m^{+}} \int_{\Omega_{j}^{+}} e^{-\lambda t}|U|_{s+j}^{2}+\sum_{j=0}^{m^{-}}+\int_{\Omega_{j}^{-}} e^{-\lambda t}|U|_{s+j}^{2}, \\
\|\| U \|_{\lambda,(0, s)}^{2} & =\sum_{j=0}^{m^{+}} \int_{\Omega_{j}^{+}} e^{-\lambda t}|U|_{(0, s+j)}^{2}+\sum_{j=0}^{m^{-}} \int_{\Omega_{j}^{-}} e^{-\lambda t}|U|_{(0, s+j)}^{2} .
\end{aligned}
$$

For $\lambda=0$, we shall simply write $\||\cdot|\|_{s}$ and $\||\cdot|\|_{(0, s)}$. We use $\|\cdot\|_{s}$ to denote the usual Sobolev $H^{s}$-norm. For $\lambda=0$, there holds for any $s \geq 0$

$$
\|U\|_{s} \leq\||| U \mid\|_{s} \leq\|U\|_{s+m}
$$

where

$$
m=\max \left\{m^{+}, m^{-}\right\} .
$$

Lemma 5.1. Let $U=(u, v)$ be a $C^{m+1}$ solution to (5.1)-(5.2) in $\Omega$ with $k^{2} \in C^{m+1}(\bar{\Omega})$ and $k \in \operatorname{Lip}(\Omega)$, with $0<k \leq 1$. Suppose (5.3) is 
satisfied. Then for any $\widetilde{U}$ in $\Omega$ with $|\widetilde{U}|_{m+2} \leq 1$, and any $\lambda \geq 1$,

$$
\begin{aligned}
& \int_{t=1} e^{-\lambda}|U|^{2}+\left.\lambda|||U|\right|_{\lambda, 0} ^{2} \\
& \leq C\left(\int_{t=0}\left(|U|_{(0, m+1)}^{2}+|G|_{m-1}^{2}\right) d x+\left.|||G|\right|_{\lambda,(0,1)} ^{2}+\left.\lambda|||G|\right|_{\lambda, 0} ^{2}\right),
\end{aligned}
$$

where $C$ is a positive constant depending only on $\theta$ in (4.7).

Proof. We only consider $t \geq 0$. The case for $t<0$ can be discussed in the same way. For simplicity, we write $m=m^{+}, \Omega=\Omega^{+}, \Omega_{j}=\Omega_{j}^{+}$ and $\Sigma_{j}=\Sigma_{j}^{+}$.

We first claim that for $j=0,1, \ldots, m$ there holds for any $\lambda>0$

$$
\begin{aligned}
& \int_{\Sigma_{j-1}} e^{-\lambda t}|U|_{j}^{2}+\lambda \int_{\Omega_{j}} e^{-\lambda t}|U|_{j}^{2} \\
& \leq C_{j}\left\{\int_{\Sigma_{j}} e^{-\lambda t}|U|_{j+1}^{2}+\int_{\Sigma_{j-1}} e^{-\lambda t}|G|_{j-1}^{2}\right. \\
& \left.\quad+\int_{\Omega_{j}} e^{-\lambda t}\left(|G|_{(0, j+1)}^{2}+\lambda|G|_{j-1}^{2}\right)\right\},
\end{aligned}
$$

where $C_{j}$ is given in Lemma 4.3. Here we denote $|G|_{-1}=0$ and $\Sigma_{-1}=$ $\{t=1\}$. For $j=0,1, \ldots, \min \left\{l_{-}, l_{+}\right\}$, we have

$$
\Omega_{j}=\left(\Omega_{j} \cap\{x \geq 0\}\right) \cup\left(\Omega_{j} \cap\{x \leq 0\} .\right.
$$

By (5.3), we simply apply Lemma 4.3 with $s=j$ to get (5.4). If $l_{-} \neq l_{+}$, we may assume $l_{+}>l_{-}$without loss of generality. Then $\Omega_{j} \subset\{x \geq 0\}$ for all $l_{-}+1 \leq j \leq l_{+}$. Therefore by means of Lemma 4.3 over the domain $\Omega_{j}$ we can still get (5.4) for all $l_{-}+1 \leq j \leq l_{+}$.

Multiplying $(5.4)_{0},(5.4)_{1}, \ldots,(5.4)_{m}$ respectively by $1, C_{0}, \ldots$, $C_{0} C_{1} \cdots C_{m-1}$, and summing them together, we obtain

$$
\begin{aligned}
& \int_{t=1} e^{-\lambda t}|U|^{2}+\lambda \sum_{j=0}^{m} \int_{\Omega_{j}} e^{-\lambda t}|U|_{j}^{2} \\
& \leq C\left\{\int_{t=0}|U|_{(0, m+1)}^{2}+\sum_{j=0}^{m-1} \int_{\Sigma_{j}} e^{-\lambda t}|G|_{j}^{2}\right. \\
& \left.\quad+\sum_{j=0}^{m} \int_{\Omega_{j}} e^{-\lambda t}\left(|G|_{(0, j+1)}^{2}+\lambda|G|_{j-1}^{2}\right)\right\},
\end{aligned}
$$


for some constant $C$ depending only on $\theta$ in (4.7) where $s=m$. In getting (5.5), we have used the remark at the end of Section 4. For integrals of $G$ over $\Sigma_{j}$, we consider

$$
\begin{aligned}
& e^{-\lambda t}|G|_{j}^{2}(x, t) \\
& \leq|G|_{j}^{2}(x, 0)+\int_{0}^{t} e^{-\lambda \tau}\left(2|G|_{j+1}(x, \tau)|G|_{j}(x, \tau)+\lambda|G|_{j}^{2}(x, \tau)\right) d \tau,
\end{aligned}
$$

which implies

$$
\begin{aligned}
\int_{\Sigma_{j}} e^{-\lambda t}|G|_{j}^{2} & \leq \int_{t=0}|G|_{j}^{2} d x+\sum_{i=j+1}^{m} \int_{\Omega_{i}} e^{-\lambda t}\left(2|G|_{j+1}|G|_{j}+\lambda|G|_{j}^{2}\right) \\
& \leq C\left(\int_{t=0}|G|_{j}^{2} d x+\lambda \sum_{i=j+1}^{m} \int_{\Omega_{i}} e^{-\lambda t}|G|_{i}^{2}\right)
\end{aligned}
$$

for some constant $C>2$. By substituting (5.6) in (5.5), we finish the proof.

q.e.d.

Next we shall establish the estimates for the higher order derivatives of solutions to (5.1)-(5.2).

In the following two results, we use $\|u\|_{s}$ to denote the $H^{s}$ norm of $u$ in $\Omega$ and $\|u(0)\|_{s}$ the $H^{s}$ norm of $u(\cdot, 0)$ in $t=0$.

We should note that both $|\| \cdot|||$ and the standard Sobolev norm $\|\cdot\|$ appear in the next result.

Lemma 5.2. Let $s \geq 0$ be an integer and $U=(u, v)$ be a $C^{m+s+1}$ solution to (5.1)-(5.2) in $\Omega$ with $k^{2} \in C^{m+s+1}(\bar{\Omega})$ and $k \in \operatorname{Lip}(\Omega)$, with $0<k \leq 1$. Suppose (5.3) is satisfied. Then for each $\widetilde{U} \in C^{m+4}(\bar{\Omega})$ with $|\widetilde{U}|_{m+4} \leq 1$, and any $\lambda \geq 1$,

$$
\begin{aligned}
& \lambda\||| U \mid\|_{\lambda, s}^{2} \\
& \leq C_{s}\left(\int_{t=0}\left(|U|_{(0, m+s+1)}^{2}+|G|_{m+s-1}^{2}\right) d x\right) \\
& \quad+C_{s}\left(\left\|| | G \left|\left\|_{\lambda,(0, s+1)}^{2}+\lambda\left|\|G \mid\|_{\lambda, s}^{2}\right)\right.\right.\right.\right. \\
& \quad+C_{s} \lambda\left(\|U\|_{s-1}^{2}+(s-2)^{+}\left(\|\widetilde{U}\|_{m+s+2}^{2}+1\right) \sup _{\Omega}\left(|U|^{2}+|G|_{m-2}^{2}\right)\right),
\end{aligned}
$$

where $C_{s}$ is a constant depending only on $\theta$ in (4.7) and $s$. 
Proof. As in the proof of Lemma 5.1, we only consider $t \geq 0$. As before, we write $m=m^{+}, \Omega=\Omega^{+}, \Omega_{j}=\Omega_{j}^{+}$and $\Sigma_{j}=\Sigma_{j}^{+}$. In the definition of $\|\mid \cdot\|$ and $\|\cdot\|$, we simply discard the the expressions in $\{t<0\}$.

$(5.7)_{0}$ is just Lemma 5.1. We may obtain $(5.7)_{1}$ and $(5.7)_{2}$ easily by modifying the following argument, if we have $|\widetilde{U}|_{m+4} \leq 1$. Now suppose that $(5.7)_{j}$ is true for all $j \leq s-1$ with $s \geq 3$. In what follows, we shall prove $(5.7)_{s}$.

An application of Lemma 5.1 to the system (4.21)-(4.22) yields

$$
\begin{aligned}
\left.\lambda||\left|U_{s}\right|\right|_{\lambda, 0} ^{2} \leq & C \int_{t=0}\left(\left|U_{s}\right|_{(0, m+1)}^{2}+\left|\partial_{x}^{s} G\right|_{m-1}^{2}\right) d x \\
& +C\left(\left|\left\|\partial _ { x } ^ { s } G \left|\left\|_{\lambda,(0,1)}^{2}+\lambda\left|\| \partial_{x}^{s} G\right|||_{\lambda, 0}^{2}\right)\right.\right.\right.\right. \\
& +C \sum_{i=1}^{2}\left(\int_{t=0}\left|g_{i s}\right|_{m-1}^{2} d x+\left(\|\left.\left|g_{i s}\right|\right|_{\lambda,(0,1)} ^{2}+\lambda||\left|g_{i s}\right|||_{\lambda, 0}^{2}\right)\right) .
\end{aligned}
$$

It suffices to estimate terms involving $g_{i s}$. From (4.23) we note

$$
\begin{aligned}
\partial^{\gamma} g_{1 s}= & \sum_{\eta+\eta^{\prime}=\gamma, i+i^{\prime}=s, i \geq 1} C_{i \eta}\left\{(i-1) \partial^{\eta} \partial_{x}^{i}\left(k^{2} \sigma\right) \partial^{\eta^{\prime}} \partial_{x}^{i^{\prime}+1} v\right\} \\
& +\sum_{\eta+\eta^{\prime}=\gamma, i+i^{\prime}=s, i \geq 1} C_{i \eta}\left\{\partial^{\eta} \partial_{x}^{i} a_{11} \partial^{\eta^{\prime}} \partial_{x}^{i^{\prime}} u+\partial^{\eta} \partial_{x}^{i} a_{12} \partial^{\eta^{\prime}} \partial_{x}^{i^{\prime}} v\right\}
\end{aligned}
$$

We write

$$
\partial^{\gamma} g_{1 s}=I_{1}+I_{2},
$$

where $I_{1}$ is the sum over all $1 \leq i+|\eta|<m+2$ and $I_{2}$ is the remaining part. Since $|\widetilde{U}|_{m+2} \leq 1$ and $\left|\eta^{\prime}\right|+i^{\prime}+1=s-i+|\gamma|-|\eta| \leq s-1+|\gamma|$, it follows that

$$
\int_{\Omega_{j}} e^{-\lambda t}\left|I_{1}\right|^{2} \leq C \int_{\Omega_{j}} e^{-\lambda t}|U|_{j+s-1}^{2} \text { as }|\gamma|=j
$$

and

$$
\int_{\Omega_{j}} e^{-\lambda t}\left|I_{1}\right|^{2} \leq C \int_{\Omega_{j}} e^{-\lambda t}|U|_{(0, j+s)}^{2} \text { as } \partial^{\gamma}=\partial_{x}^{j+1}
$$


For terms in $I_{2}$, we shall use an interpolation inequality. To do this, we first need to extend the integral to the whole region. Under the present circumstance, $\left|\eta^{\prime}\right|+i^{\prime}+1 \leq s+|\gamma|-m-2 \leq s-2$ as $|\gamma| \leq m$. Hence, an interpolation inequality yields, as $|\gamma|=j$,

$$
\int_{\Omega_{j}} e^{-\lambda t}\left|I_{2}\right|^{2} \leq \int_{0}^{1} \int_{-\infty}^{\infty}\left|I_{2}\right|^{2} \leq C\left(\left.|| U\right|_{s-2} ^{2}+\left(\|\left.\widetilde{U}\right|_{m+s+1} ^{2}+1\right)|U|_{\infty}^{2}\right),
$$

and as $\partial^{\alpha}=\partial_{x}^{j+1}$,

$$
\int_{\Omega_{j}} e^{-\lambda t}\left|I_{2}\right|^{2} \leq C\left(\left.|| U\right|_{s-1} ^{2}+\left(\left.|| \widetilde{U}\right|_{m+s+2} ^{2}+1\right)|U|_{\infty}^{2}\right) .
$$

Similarly we get the same estimates for $g_{2 s}$. It remains to estimate

$$
Q_{s}=\sum_{i=1}^{2} \int_{t=0}\left|g_{i s}\right|_{m-1}^{2} d x
$$

In (5.9), we consider $|\gamma| \leq m-1$. We shall use the interpolation inequality along $t=0$. The maximal order for $t$ derivative is $m-1$. In (5.9), we shall keep the $t$-derivative of $a_{i j}$ and $k^{2} \sigma$. We shall use the system (5.1)-(5.2) repeatedly to replace the $t$-derivatives of $u$ and $v$ by their $x$-derivatives and also the (mixed) derivatives of $g_{1}$ and $g_{2}$. The maximal order of $t$-derivatives of $g_{1}$ and $g_{2}$ is $m-2$. In the resulted identity, we treat $t$-derivatives as individual functions. Now we may apply the interpolation inequality to get

$$
\begin{aligned}
Q_{s} \leq & C\left(\int_{t=0}|U|_{(0, m+s-1)}^{2}+\left(\int_{t=0}|\widetilde{U}|_{s+m}^{2} d x+1\right) \sup _{t=0}|U|^{2}\right) \\
& +C\left(\int_{t=0}|G|_{m+s-2}^{2}+\left(\int_{t=0}|\widetilde{U}|_{s+m}^{2} d x+1\right) \sup _{t=0}|G|_{m-2}^{2}\right) .
\end{aligned}
$$

We may use the trace theorem to replace the integrals of $\widetilde{U}$ along $t=0$ by integrals over $\Omega$. Therefore we obtain

$$
\begin{aligned}
Q_{s} \leq & C \int_{t=0}\left(|U|_{(0, m+s-1)}^{2}+|G|_{m+s-2}^{2}\right) d x \\
& +C\left(\|\widetilde{U}\|_{s+m+1}^{2}+1\right) \sup _{t=0}\left(|U|^{2}+|G|_{m-2}^{2}\right) .
\end{aligned}
$$


Now combining (5.8) with (5.11)-(5.14) we have

$$
\begin{aligned}
& \lambda\left|\left\|U_{s}|\||_{\lambda, 0}^{2} \leq C \int_{t=0}\left(|U|_{(0, m+s+1)}^{2}+|G|_{m+s-1}^{2}\right) d x\right.\right. \\
& +C\left(\left\||| G||_{\lambda,(0, s+1)}^{2}+\lambda\left|\|G \mid\|_{\lambda, s}^{2}\right)+C\left(\left.\left|\left\|U_{s}||_{\lambda, 0}^{2}+\lambda\right\|\right||U|\right|_{\lambda, s-1} ^{2}\right)\right.\right. \\
& +C \lambda\left(\|U\|_{s-1}^{2}+\left(\|\left.\widetilde{U}\right|_{m+s+2} ^{2}+1\right) \sup _{\Omega}\left(|U|^{2}+|G|_{m-2}^{2}\right)\right) .
\end{aligned}
$$

We move the term $\left\|\left|U_{s}\right|\right\|_{\lambda, 0}^{2}$ in the second line in (5.15) to the left side and rename $\lambda$. Such an inequality illustrates the estimate of $\partial_{x}^{s} U$. For other $s$-derivatives, we proceed as in the proof of Lemma 4.3. Then we get

$$
\begin{aligned}
& \left.\lambda|\| U|\right|_{\lambda, s} ^{2} \leq C\left(\int_{t=0}\left(|U|_{(0, m+s+1)}^{2}+|G|_{m+s-1}^{2}\right) d x\right) \\
& +C\left(\left\||| G\left|\left\|_{\lambda,(0, s+1)}^{2}+\left.\lambda|\| G|\right|_{\lambda, s} ^{2}\right)+C \lambda\right|\right\| U \mid \|_{\lambda, s-1}^{2}\right. \\
& \quad+C \lambda\left(\|U\|_{s-1}^{2}+\left(\|\left.\widetilde{U}\right|_{m+s+2} ^{2}+1\right) \sup _{\Omega}\left(|U|^{2}+|G|_{m-2}^{2}\right)\right) .
\end{aligned}
$$

Therefore, we obtain $(5.7)_{s}$ by the hypothesis of induction. q.e.d.

Now we state Lemma 5.2 in terms of the usual Sobolev norms.

Theorem 5.3. Let $s \geq 0$ be an integer and $U=(u, v)$ be $a C^{m+s+1}$ solution to (5.1)-(5.2) in $\Omega$, with $k^{2} \in C^{m+s+1}(\bar{\Omega})$ and $k \in \operatorname{Lip}(\Omega)$, $0<k \leq 1$. Suppose (5.3) is satisfied. Then for each $\widetilde{U} \in C^{m+4}(\bar{\Omega})$ with $|\widetilde{U}|_{m+4} \leq 1$, we have

$$
\begin{aligned}
\|U\|_{s} \leq C_{s}\left(\|U(0)\|_{m+s+1}\right. & +\|G\|_{s+m+1} \\
& \left.+(s-2)^{+}\left(\|\widetilde{U}\|_{m+s+2}+1\right)\|G\|_{m+3}\right)
\end{aligned}
$$

where $C_{s}$ is a constant depending only on $\theta$ in (4.7) and $s$. Moreover, if $U=0$ at $t=0$, we have

$$
\|U\|_{s} \leq C_{s}\left(\|G\|_{s+m+1}+(s-2)^{+}\left(\|\widetilde{U}\|_{m+s+2}+1\right)\|G\|_{m+3}\right) .
$$

Proof. By fixing a $\lambda \geq 1$ and using the usual Sobolev norm in (5.7), we obtain

$$
\begin{aligned}
\|U\|_{s}^{2} \leq & C_{s}\left(\int_{t=0}\left(|U|_{(0, m+s+1)}^{2}+|G|_{m+s-1}^{2}\right) d x+\|G\|_{s+m+1}^{2}\right) \\
& +C_{s}\left(\|U\|_{s-1}^{2}+(s-2)^{+}\left(\|\widetilde{U}\|_{m+s+2}^{2}+1\right) \sup _{\Omega}\left(|U|^{2}+|G|_{m-2}^{2}\right)\right) .
\end{aligned}
$$


This holds for any $s \geq 0$. By induction on $s$, we have

$$
\begin{aligned}
\|U\|_{s}^{2} \leq & C_{s}\left(\int_{t=0}\left(|U|_{(0, m+s+1)}^{2}+|G|_{m+s-1}^{2}\right) d x+\|G\|_{s+m+1}^{2}\right) \\
& +C_{s}(s-2)^{+}\left(\|\widetilde{U}\|_{m+s+2}^{2}+1\right) \sup _{\Omega}\left(|U|^{2}+|G|_{m-2}^{2}\right) .
\end{aligned}
$$

Then (5.17) follows from the trace theorem and Sobolev embedding.

q.e.d.

As (5.17) and (5.18) show, there is a loss of derivatives in the solution. This is due to the degeneracy of our system. The loss is related to how many times $\partial_{t} k$ changes its sign.

\section{The proof for the main theorem}

Now we shall use the Nash-Moser-Hörmander procedure to prove the existence of sufficiently smooth local isometric embedding for metrics satisfying the assumption $(\mathrm{A})$. We set

$$
\Omega=\{(x, t) ; x \in \mathbf{R}, t \in(-1,1)\} .
$$

Here $\{(x, t)\}$ is the coordinate system we used in Section 2. In particular, the Gaussian curvature $K$ satisfies the assumption (A) in $\{(x, t)\}$.

For any natural number $q$, by a standard technique, we have a linear operator

$$
P: H^{q}(\Omega) \longrightarrow H^{q}\left(\mathbf{R}^{2}\right) \text { such that } P(\widetilde{U})=\widetilde{U} \text { in } \Omega,
$$

with the property

$$
\frac{1}{C}\|\widetilde{U}\|_{H k_{(\Omega)}} \leq\|P(\widetilde{U})\|_{H^{k}\left(\mathbf{R}^{2}\right)} \leq C\|\widetilde{U}\|_{H k_{(\Omega)}},
$$

for some universal constant $C$ and all $k=0,1,2, \ldots, q$. For details, see [8] or [6]. Moreover, if $q \geq m+6$, we may assume this operator has the additional properties

$$
P: C^{m+4}(\bar{\Omega}) \longrightarrow C^{m+4}\left(\mathbf{R}^{2}\right) \text { such that } P(\widetilde{U})=\widetilde{U} \text { in } \Omega,
$$

and

$$
\frac{1}{C}|\widetilde{U}|_{C^{m+4}(\bar{\Omega})} \leq|P(\widetilde{U})|_{C^{m+4}\left(\mathbf{R}^{2}\right)} \leq C|\widetilde{U}|_{C^{m+4}(\bar{\Omega})} .
$$


Consider also the smoothing operator $J_{\delta}: H_{-\infty}\left(\mathbf{R}^{2}\right) \longrightarrow H^{\infty}\left(\mathbf{R}^{2}\right)$ for $\delta \in(0,1)$ as used in [6]. Then we have

$$
\begin{aligned}
& \left\|J_{\delta} u\right\|_{s_{1}} \leq C_{s_{1} s_{2}}\left(\frac{1}{\delta}\right)^{s_{1}-s_{2}}\|u\|_{s_{2}} \text { as } u \in H^{s_{1}}\left(\mathbf{R}^{2}\right) \text { and } s_{1} \geq s_{2} \\
& \left\|u-J_{\delta} u\right\|_{s_{2}} \leq C_{s_{1} s_{2}} \delta^{s_{1}-s_{2}}\|u\|_{s_{1}} \text { as } u \in H^{s_{1}}\left(\mathbf{R}^{2}\right) \text { and } s_{1} \geq s_{2}
\end{aligned}
$$

and

$$
\left\|u-J_{\delta} u\right\|_{s_{1}} \longrightarrow 0 \text { as } \delta \longrightarrow 0 .
$$

Next we proceed to solve (2.8) by the Nash-Moser-Hörmander procedure. Suppose

$$
\widetilde{U}_{0}=0,
$$

and

$$
\widetilde{U}_{n+1}=\widetilde{U}_{n}+U_{n}, \text { for } n=0,1, \ldots,
$$

where $U_{n}$ is a sufficiently smooth solution to the Cauchy problem for the regularized system

$$
\begin{aligned}
& \partial_{t} u+\frac{1}{2}\left(u_{0}+\varepsilon \widetilde{u}\right) \partial_{x} u-\left(k^{2}+d_{n}\right)\left(v_{0}+\varepsilon \widetilde{v}\right) \partial_{x} v+a_{11} u+a_{12} v=\chi\left(g_{n}\right)_{1}, \\
& (6.10) \quad \partial_{t} v+\frac{1}{2}\left(u_{0}+\varepsilon \widetilde{u}\right) \partial_{x} v-\left(v_{0}+\varepsilon \widetilde{v}\right) \partial_{x} u+a_{21} u+a_{22} v=\chi\left(g_{n}\right)_{2}
\end{aligned}
$$

with

$$
u(x, 0)=0, \quad v(x, 0)=0 .
$$

In (6.9)-(6.10), $\chi$ is a cutoff function equal to zero as $|x| \geq 1 / 2$ and equal to 1 as $|x| \leq 1 / 4, k^{2}=-K, a_{i j}$ is as in (2.12),

$$
\begin{gathered}
\widetilde{U}=J_{n}\left(P \widetilde{U}_{n}\right), \quad \text { with } J_{n}=J_{\delta_{n}} \text { and } \delta_{n}=2^{-n}, \\
d_{n}=\sup _{|x| \leq 1 / 8}\left\{\left|F\left(\widetilde{U}_{n}\right)(x, t)\right|\right\},
\end{gathered}
$$

and $g_{n}$ is determined by the following procedure. The key point is to make the accumulative error not increasing as $n$ increases. More precisely, we set with $J_{-1}=0$

$$
g_{n}=\left(J_{n-1}-J_{n}\right) P F(0)+J_{n-1} P E_{n-1}-J_{n} P E_{n},
$$


where

$$
E_{n}=\sum_{i=0}^{n-1} e_{i}, E_{0}=0, e_{n}=e_{n}^{\prime}+e_{n}^{\prime \prime}+e_{n}^{\prime \prime \prime},
$$

and

$$
\begin{aligned}
e_{n}^{\prime} & =\left(F^{\prime}\left(\widetilde{U}_{n}\right)-F^{\prime}\left(J_{n}\left(P \widetilde{U}_{n}\right)\right)\right) U_{n}, \\
e_{n}^{\prime \prime} & =\left(\begin{array}{c}
d_{n}\left(v_{0}+\varepsilon J_{n}(P \widetilde{v})\right) \partial_{x} v_{n} \\
0
\end{array}\right), \\
e_{n}^{\prime \prime \prime} & =F\left(\widetilde{U}_{n+1}\right)-F\left(\widetilde{U}_{n}\right)-F^{\prime}\left(\widetilde{U}_{n}\right) U_{n},
\end{aligned}
$$

and $F^{\prime}\left(\widetilde{U}_{n}\right)$ is the linearized operator of $F$ at $\widetilde{U}_{n}$. Consequently, we have

$$
\begin{aligned}
F\left(\widetilde{U}_{n+1}\right) & =F\left(\widetilde{U}_{n}\right)+\chi g_{n}+e_{n}^{\prime}+e_{n}^{\prime \prime}+e_{n}^{\prime \prime \prime} \\
& =(1-\chi)\left(F(0)+E_{n}\right)+e_{n}+\chi\left(I-J_{n}\right) P\left(F(0)+E_{n}\right) .
\end{aligned}
$$

Let $m=\max \left\{l_{+}, l_{-}\right\}$be the integer introduced in the previous section. For any $r>2 m+12$, we fix $q=[r]-3$ and $p=r-m-5-r_{0}$, where $r_{0}=\bar{\varepsilon}+r-[r] \in(0,1)$ by choosing an appropriate $\bar{\varepsilon}>0$. Hence $p>m+6$ and $p \leq[r]-m-5$, since $r>2 m+12$.

In the next result, we write $U_{-1}=0, T_{-1}=I$.

Lemma 6.1. Suppose $r>2 m+12$. Then there is a positive constant $\varepsilon_{*}$ such that if

$$
\|F(0)\|_{p+m+1}=\mu^{2} \leq \varepsilon_{*},
$$

then for all nonnegative integers $s$,

$$
\begin{aligned}
& \left\|U_{j-1}\right\|_{s} \leq \mu 2^{(j-1)(s-p)}, \quad 0 \leq s \leq[r]-3, \\
& \left\{\begin{array}{l}
\left\|\widetilde{U}_{j}\right\|_{s},\left\|J_{j} P \widetilde{U}_{j}\right\|_{s} \leq C_{1} \mu 2^{j(s-p)^{+}}, \quad 0 \leq s \leq[r]-3, \\
\left\|\left(I-J_{j}\right) P \widetilde{U}_{j}\right\|_{s} \leq C_{2} \mu 2^{(j-1)(s-p)}, \quad 0 \leq s
\end{array}\right. \\
& \left\|e_{j-1}\right\|_{s+m+1} \leq C_{3} \mu^{2} 2^{(j-1)(s-p)}, \quad-m-1 \leq s \leq[r]-m-5, \\
& \left\|g_{j}\right\|_{s+m+1} \leq C_{4} \mu^{2} 2^{j(s-p)}, \quad-m-1 \leq s \leq[r]-3,
\end{aligned}
$$

and

$$
\left|d_{j}\right| \leq \mu 2^{j(2-p)},
$$

for some positive constants $C_{1}, C_{2}, C_{3}$ and $C_{4}$ independent of $j$ and $\mu$. 
Proof. We denote $\varepsilon=\mu^{2}$. First introduce a transformation of coordinates

$$
T_{n}: \bar{\Omega} \ni(x, t) \mapsto(\xi, \eta)=\left(\psi_{n}(x, t), t\right) \in \bar{\Omega}
$$

where

$$
\psi_{n} \text { is a sufficiently smooth solution to }(2.15) \text { with } \widetilde{u}=J_{\delta_{n}} \widetilde{u}_{n} \text {. }
$$

In the sequel we always assume that $\varepsilon$ is so small that Lemma 2.1 and Lemma 2.2 hold. Therefore the problem (6.9)-(6.10) is reduced to

$$
\begin{aligned}
& L_{1}(u, v) \equiv \partial_{\eta} u_{n}-\left(k^{2}+d_{n}\right) \sigma \partial_{\xi} v_{n}+a_{11} u_{n}+a_{12} v_{n}=\chi\left(g_{n}\right)_{1}, \\
& L_{2}(u, v) \equiv \partial_{\eta} v_{n}-\sigma \partial_{\xi} u_{n}+a_{21} u_{n}+a_{22} v_{n}=\chi\left(g_{n}\right)_{2},
\end{aligned}
$$

where $\sigma$ is given by $(2.20)$, with $\widetilde{v}=J_{\delta_{n}} \widetilde{v}_{n}$. For convenience, we denote $U_{n} \circ T_{n}^{-1}, g_{n} \circ T_{n}^{-1}, \ldots$ by $U_{n}, g_{n}, \ldots$ This is a strictly hyperbolic system with $C^{r-3}$ coefficients if $d_{n}>0$, and hence always admits a solution in $H^{q}(\Omega), q=[r]-3$, to its Cauchy problem with (6.11). Moreover, by the property of the propagation at finite speed, the above solution is of compact support in $\xi$. More precisely, its support is contained in

$$
\begin{gathered}
\left\{(\xi, \eta) ;-\frac{1}{2}-|\eta| \sup \left|\sigma \sqrt{k^{2}+d_{n}}\right| \leq \xi \leq \frac{1}{2}+|\eta| \sup \left|\sigma \sqrt{k^{2}+d_{n}}\right|\right\} \\
\subset[-1,1] \times[-1,1]
\end{gathered}
$$

if $\varepsilon$ is small. After pulling back to the original coordinates it is still in $H^{q}(\Omega)$ and of compact support in $x$. By $(2.17)$, we have

$$
\partial_{t} k+\frac{1}{2}\left(\hat{u}+\varepsilon J_{n}\left(P \widetilde{u}_{n}\right)\right) \partial_{x} k=\partial_{\eta} k .
$$

By the extended assumption (A), all the hypotheses in Theorem 5.3 are satisfied as long as

$$
\left|J_{n}\left(P \widetilde{U}_{n}\right)\right|_{m+4} \leq 1
$$

Therefore, by Theorem 5.3 we obtain for $s \leq[r]-3$

$$
\left\|U_{n}\right\|_{s} \leq C_{s}\left(\left\|\chi g_{n}\right\|_{s+m+1}+(s-2)^{+}\left(\left\|J_{n} P \widetilde{U}_{n}\right\|_{m+s+2}+1\right)\left\|\chi g_{n}\right\|_{m+3}\right) \text {. }
$$

Such an estimate holds in the coordinate system $\{(\xi, \eta)\}$. By Lemma 2.2 and the interpolation inequality, it is easy to see that it also holds in $\{(x, t)\}$. 
In what follows, we shall prove Lemma 6.1 by induction on $j$.

If $j=0,(6.19)_{0^{-}}(6.22)_{0}$ are trivial for any positive constant $\mu$. Note $\widetilde{U}_{0}=e_{-1}=0$ and $g_{0}=-J_{0}(P F(0))$. Then $(6.21)_{0}$ comes from (6.5) and (6.6). Also $d_{0}=\sup |F(0)| \leq C \mu^{2} \leq \mu$, if $C \mu \leq 1$.

The proof for $(6.18)_{1}$. By $(6.24)$, we have

$$
\begin{aligned}
\left\|U_{0}\right\|_{s} & \leq C\left(\left\|\chi g_{0}\right\|_{s+m+1}+(s-2)^{+}\left\|\chi g_{0}\right\|_{m+3}\left(\left\|\widetilde{U}_{0}\right\|_{s+m+2}+1\right)\right) \\
& \leq C C_{4} \mu^{2} \leq \mu
\end{aligned}
$$

if $C C_{4} \mu \leq 1$. This proves $(6.18)_{1}$.

Now let us assume $(6.18)_{n}$ and $(6.19)_{n-1^{-}}(6.22)_{n-1}$ are valid for all $n \geq 1$. We shall prove $(6.19)_{n^{-}}(6.22)_{n}$ and $(6.18)_{n+1}$ successively.

The proof for $(6.19)_{n}$. First note

$$
\begin{aligned}
\left\|\widetilde{U}_{n}\right\|_{s} & \leq \sum_{j=0}^{n-1}\left\|U_{j}\right\|_{s} \leq \mu \sum_{j=0}^{n-1} 2^{j(s-p)} \\
& \leq \begin{cases}\mu 2^{n(s-p)} \sum_{j=1}^{\infty}\left(\frac{1}{2}\right)^{j(s-p)} & \text { if } s>p, \\
\mu \sum_{j=0}^{\infty}\left(\frac{1}{2}\right)^{j\{p-[p]\}} & \text { if } s<p .\end{cases}
\end{aligned}
$$

This implies the first part of $(6.19)_{n}$ if we take $C_{1}=1 /\left\{1-2^{[p]-p}\right\}$. The second part of $(6.19)_{n}$ is the immediate consequence of (6.5). And (6.6) yields

$$
\left\|\left(I-J_{n}\right) P \widetilde{U}_{n}\right\|_{s} \leq C_{5} \mu 2^{n(s-q)}\left\|P \widetilde{U}_{n}\right\|_{q} \leq C_{1} C_{5} \mu 2^{n(s-p)} .
$$

Hence we get the last part of $(6.19)_{n}$ by taking $C_{2}=C_{1} C_{5}$.

Throughout the following argument we always assume $\varepsilon_{*}$ so small that

$$
\left|\widetilde{U}_{n}\right|_{m+4},\left|J_{n} P \widetilde{U}_{n}\right|_{m+4} \leq 1 .
$$

This is possible because $p=r-m-5-r_{0}>m+6$ and

$$
\left|\widetilde{U}_{n}\right|_{m+4} \leq\left. C|| \widetilde{U}_{n}\right|_{m+6} \leq C C_{1} \mu \leq C C_{1} \sqrt{\varepsilon_{*}} .
$$

The proof for $(6.20)_{n}$. We shall estimate each three terms in the sum $e_{n-1}=e_{n-1}^{\prime}+e_{n-1}^{\prime \prime}+e_{n-1}^{\prime \prime \prime}$. First by (6.14)

$$
e_{n-1}^{\prime}=\left(F^{\prime}\left(\widetilde{U}_{n-1}\right)-F^{\prime}\left(J_{n-1} P \widetilde{U}_{n-1}\right)\right) U_{n-1},
$$


we have from (2.10)-(2.11)

$$
\begin{aligned}
e_{n-1}^{\prime}= & \left.\left.\int_{0}^{1} F^{\prime \prime}\left(\widetilde{U}_{n-1}+\lambda\left(I-J_{n-1} P\right) \widetilde{U}_{n-1}\right)\right)\left(U_{n-1},\left(I-J_{n-1} P\right) \widetilde{U}_{n-1}\right)\right) d \lambda \\
= & \frac{1}{2} \varepsilon\left(\begin{array}{cc}
\left(I-J_{n-1}\right) P \widetilde{u}_{n-1} & K\left(I-J_{n-1}\right) P \widetilde{v}_{n-1} \\
-\left(I-J_{n-1}\right) P \widetilde{v}_{n-1} & \left(I-J_{n-1}\right) P \widetilde{u}_{n-1}
\end{array}\right) \partial_{x} U_{n-1} \\
& +\left(\begin{array}{ll}
{\left[a_{11}\right]} & {\left[a_{12}\right]} \\
{\left[a_{21}\right]} & {\left[a_{22}\right]}
\end{array}\right) U_{n-1},
\end{aligned}
$$

where $\left[a_{i j}\right]$ is given by

$$
\left[a_{i j}\right]=\int_{0}^{1} \frac{\partial a_{i j}}{\partial(U)}\left(\widetilde{U}_{n-1}+\lambda\left(I-J_{n-1} P\right) \widetilde{U}_{n-1}\right)\left[\left(I-J_{n-1} P\right) \widetilde{U}_{n-1}\right] d \lambda
$$

By the interpolation inequality as done in proving (5.12) and (5.13), we get for all $-m-1 \leq s \leq[r]-m-5$,

$$
\begin{aligned}
&\left\|e_{n-1}^{\prime}\right\|_{s+m+1} \\
& \leq C_{6}\left\|U_{n-1}\right\|_{s+m+2}\left\|\left(I-J_{n-1}\right) P \widetilde{U}_{n-1}\right\|_{2} \\
&+C_{6}\left\|U_{n-1}\right\|_{3}\left\|\left(I-J_{n-1}\right) P \widetilde{U}_{n-1}\right\|_{s+m+1} \\
&+C_{6}\left\|U_{n-1}\right\|_{3}\left\|\left(I-J_{n-1}\right) P \widetilde{U}_{n-1}\right\|_{2}\left(\left\|\widetilde{U}_{n-1}\right\|_{s+m+1}+1\right) \\
& \leq 2 C_{6} C_{1} C_{2} \mu^{2}\left(2^{(n-1)(s+m+4-2 p)}+2^{(n-1)\left[5-2 p+(s+m+1-p)^{+}\right]}\right) \\
& \leq 4 C_{6} C_{1} C_{2} \mu^{2} 2^{(n-1)(s-p)},
\end{aligned}
$$

if $p>m+6$. Next, recall from (6.16)

$$
\begin{aligned}
& e_{n-1}^{\prime \prime \prime}=\int_{0}^{1}\left[F^{\prime}\left(\widetilde{U}_{n-1}+\lambda U_{n-1}\right)-F^{\prime}\left(\widetilde{U}_{n-1}\right)\right] U_{n-1} d \lambda \\
& =\left(\begin{array}{c}
u_{n-1} \partial_{x} u_{n-1}-\frac{1}{2} k^{2} v_{n-1} \partial_{x} v_{n-1} \\
u_{n-1} \partial_{x} v_{n-1}-\frac{1}{2} v_{n-1} \partial_{x} u_{n-1}
\end{array}\right)+\left(\begin{array}{ll}
{\left[\left[a_{11}\right]\right],} & {\left[\left[a_{12}\right]\right]} \\
{\left[\left[a_{21}\right]\right],} & {\left[\left[a_{22}\right]\right]}
\end{array}\right) U_{n-1},
\end{aligned}
$$

where

$$
\left[\left[a_{i j}\right]\right]=\int_{0}^{1} \int_{0}^{1} \frac{\partial a_{i j}}{\partial U}\left(\widetilde{U}_{n-1}+t \lambda U_{n-1}\right) U_{n-1} d \lambda d t .
$$

In a similar argument we can also derive $\left\|e_{n-1}^{\prime \prime \prime}\right\|_{s}$ controlled by the 
right-hand side of (6.25). Analogously, we have by (6.15)

$$
\begin{aligned}
& \left\|e_{n-1}^{\prime \prime}\right\|_{s+m+1} \\
& \leq C_{7} d_{n-1}\left\|\partial_{x} v_{n-1}\right\|_{s+m+1}\left(\left\|J_{n-1} P \widetilde{U}_{n-1}\right\|_{2}+1\right) \\
& \quad+C_{7} d_{n-1}\left\|\partial_{x} v_{n-1}\right\|_{2}\left(\left\|\widetilde{U}_{n-1}\right\|_{s+m+1}+1\right) \\
& \leq C_{7} C_{1} C_{2} \mu^{2}\left(2^{(n-1)(s+m+4-2 p)}+2^{(n-1)\left[5-2 p+(s+m+1-p)^{+}\right]}\right) \\
& \leq 2 C_{7} C_{1} C_{2} \mu^{2} 2^{(n-1)(s-p)}
\end{aligned}
$$

since $p>m+6$. Combining the above estimates soon gives $(6.20)_{n}$ if we take $C_{3}=4 C_{1} C_{2}\left(C_{6}+C_{7}\right)$.

The proof for $(6.21)_{n}$. Note

$$
\begin{aligned}
\left\|E_{n-1}\right\|_{[r]-4} & \leq \sum_{j=0}^{n-2}\left\|e_{j}\right\|_{[r]-4} \leq C_{3} \mu^{2} \sum_{j=0}^{n-2} 2^{j([r]-m-5-p)} \\
& \leq 2 C_{3} \mu^{2} 2^{(n-2)([r]-m-5-p)}
\end{aligned}
$$

since $[r]-m-5-p=r_{0}-r+[r]=\bar{\varepsilon}>0$ by the choice of $\bar{\varepsilon}$. With the aid of (6.5) or (6.6), it implies

$$
\begin{aligned}
\left\|\left(J_{n-1}-J_{n}\right) E_{n-1}\right\|_{s+m+1} & \leq C_{8} 2^{(n-1)[s+m+1-([r]-4)]}\left\|E_{n-1}\right\|_{[r]-4} \\
& \leq C_{8} C_{3} \mu^{2} 2^{(n-1)(s-p)}
\end{aligned}
$$

for all $-m-1 \leq s \leq[r]-3$. Now we have by (6.13)

$$
\begin{aligned}
\left\|g_{n}\right\|_{s+m+1} \leq & \left\|\left(J_{n-1}-J_{n}\right) P F(0)\right\|_{s+m+1} \\
& +\left\|\left(J_{n-1}-J_{n}\right) P E_{n-1}\right\|_{s+m+1}+\left\|J_{n} P e_{n-1}\right\|_{s+m+1} \\
\leq & C_{8} 2^{n(s-p)}\|F(0)\|_{p+m+1}+C_{3} C \mu^{2} 2^{n(s-p)}+C C_{3} \mu^{2} 2^{n(s-p)} \\
\leq & C\left(C_{8}+2 C_{3}\right) \mu^{2} 2^{n(s-p)}
\end{aligned}
$$

since $p+m+1=r-4-r_{0}>r-5>0$. This implies $(6.21)_{n}$ if we take $C_{4}=C\left(C_{8}+2 C_{3}\right)$.

The proof for $(6.22)_{n}$. By the definition of $d_{n}$ in (6.12) and (6.17), we have

$$
\begin{aligned}
d_{n} & \leq \sup \left|e_{n-1}\right|+\sup \left|\left(I-J_{n-1}\right) P\left(F(0)+E_{n-1}\right)\right| \\
& \leq 2 C C_{3}\left(\mu^{2}+\|F(0)\|_{2}\right) 2^{(n-1)(2-p)} \leq 4 C^{\prime} C_{3} \mu^{2} 2^{n(2-p)},
\end{aligned}
$$

for some constant $C^{\prime}$. This implies $(6.22)_{n}$ if $4 C^{\prime} C_{3} \mu \leq 1$. 
The proof for $(6.18)_{n+1}$. By (6.24), we have

$$
\begin{aligned}
\left\|U_{n}\right\|_{s} & \leq C_{9}\left(\left\|\chi g_{n}\right\|_{s+m+1}+(s-2)^{+}\left\|\chi g_{n}\right\|_{m+3}\left\|J_{n} P \widetilde{U}_{n}\right\|_{s+m+2}\right) \\
& \leq C_{9}\left(C_{4}+C_{2}\right) \mu^{2}\left(2^{n(s-p)}+2^{n\left[(-4-p)+(s+m+2-p)^{+}\right]}(s-2)^{+}\right) \\
& \leq 2 C_{9}\left(C_{4}+C_{2}\right) \mu^{2} 2^{n(s-p)},
\end{aligned}
$$

since $p>m+6$. Then $(6.18)_{n+1}$ is proved if $\mu$ is so small that $2 C_{9}\left(C_{3}+\right.$ $\left.C_{4}\right) \mu \leq 1$. We have completed the proof of the present lemma. q.e.d.

Now we finish the proof of our main result, Theorem 1.1.

Proof. From the assumption (A) it is easy to see $m=\max \left\{l_{+}, l_{-}\right\} \leq$ $l$. So we take $q=[r]-3$ and $r_{0}=\bar{\varepsilon}+r-[r] \in(0,1)$ with $\bar{\varepsilon}>0$, such that $p=r-l-5-r_{0}>l+6$ and $p<q-l-2=[r]-5-l$. This is possible by the assumption $r>2 l+12$. Therefore all the requirements in Lemma 6.1 are fulfilled. By (6.18), it follows

$$
\sum\left\|U_{j}\right\|_{[p]} \leq \mu \sum 2^{-(p-[p]) j}<\infty
$$

This implies, by the definition of $\widetilde{U}_{j}$ in $(6.8)$, that $\widetilde{U}_{j} \rightarrow \widetilde{U}$ in $H^{[p]}(\Omega)$ as well as in $C^{[p]-2}(\bar{\Omega})$. Moreover, we have

$$
F(\widetilde{U})=\lim _{j \rightarrow \infty} F\left(\widetilde{U}_{j}\right) \text { in } C^{[p]-3}
$$

and for $|x| \leq 1 / 8$

$$
|F(\widetilde{U})| \leq \lim _{j \rightarrow \infty} d_{j}=0
$$

So $\widetilde{U}=(\widetilde{u}, \widetilde{v})$ is a $C^{[p]-2}$ solution to $(2.8)$ for $|x| \leq 1 / 8$ and $|t| \leq 1$. In fact, $\widetilde{U}$ is a $C^{p-\delta-2}$ solution for small $\delta>0$. To see this, we use the interpolation inequality to get by $(6.18)$

$$
\left\|U_{j}\right\|_{p-\delta} \leq C|| U_{j}\left\|_{[p]}^{[p]+1-p+\delta}\right\| U_{j} \|_{[p]+1}^{p-[p]-\delta} \leq C \mu^{-j \delta} .
$$

By (2.4) and (2.13) we can find $L, M$ and $N$ in $C^{p-2-\delta}$ satisfying the Gauss-Codazzi system near $x=t=0$. Finally, by the Fundamental Theorem on Differential Geometry we can find the expected $C^{p-\delta} \subset$ $C^{r-6-l}$ isometric embedding near $x=t=0$, by choosing $\delta$ small. This ends the proof for Theorem 1.1.

q.e.d. 


\section{Remarks on the hypothesis}

In this section, we shall discuss the hypothesis in the main result. Let us recall the assumption (A). We make a slight change in the presentation.

For a fixed positive integer $l$, some small constant $\delta>0$ and a pair of orthogonal unit vectors $(\eta, \nu)$, the following holds in a neighborhood $N$ of 0: for any $C^{1}$ function $u$ with $|u|_{C^{1}(N)}<\delta$, there exist finitely many curves $\gamma_{i}, i=1, \ldots, l$, meeting at the origin nontangentially, such that $N$ is separated into finitely many subdomains by $\gamma_{i}, i=1, \ldots, l$, and $\partial_{\nu} K+u \partial_{\eta} K$ changes its sign only across $\gamma_{1}, \ldots, \gamma_{l}$. Moreover, none of the curves $\gamma_{i}, i=1, \ldots, l$, is tangent to $\eta$ and $\nu$.

As mentioned in the introduction, the assumption (A) has a simple geometric interpretation. It means that the zero set of $\partial_{\nu} K$ consists of finitely many curves meeting nontangentially at the origin and that such a picture is preserved under the perturbation of the directional derivatives of $K$.

Now we give several classes of Gaussian curvature satisfying (A).

\subsection{The Gaussian curvature vanishes at finite orders and leading polynomials are decisive}

If $K$ vanishes at a finite order, then $u \partial_{\eta} K$ is a small perturbation of the lowest order of $\partial_{\nu} K$. We let

$$
K=-P(x, t)+o\left(|(x, t)|^{n}\right),
$$

where $P$ is a homogeneous polynomial of an even degree $n$. We know the zero set of $\partial_{\nu} P$ consists of finitely many straight lines intersecting at 0 . We need to check whether this picture is stable.

Lemma 7.1. Suppose $K$ is given in a neighborhood $N$ of 0 by

$$
K=-\left(\nu_{1} \cdot(x, t)\right)^{2} \cdots\left(\nu_{k} \cdot(x, t)\right)^{2} Q(x, t)+\text { h.o.t. }
$$

where $Q(x, t)$ is a homogeneous polynomial with $Q(x, t)>0$ for $(x, t) \neq$ 0 and $\nu_{1}, \ldots, \nu_{k}$ are distinct unit vectors. Then $K$ satisfies the assumption (A).

Remark. Note $\nu_{i} \cdot(x, t)$ is a homogeneous linear function for each $i=1, \ldots, k$. Hence (7.1) means we do not allow linear factors with the higher order in the leading polynomials. On the other hand, linear factors may not be present at all. 
Proof. Let $P$ be the homogeneous polynomial of an even degree $n$ given by

$$
P(x, t)=\left(\nu_{1} \cdot(x, t)\right)^{2} \cdots\left(\nu_{k} \cdot(x, t)\right)^{2} Q(x, t) .
$$

We may write

$$
K=-P(x, t)+R(x, t)=-P(x, t)+o\left(|(x, t)|^{n}\right),
$$

where $R$ consists of higher order terms.

We first claim there exists a unit vector $\nu$ such that

$$
\partial_{\nu} P(t, x)=\left(\widetilde{\nu}_{1} \cdot(x, t)\right) \cdots\left(\widetilde{\nu}_{l} \cdot(x, t)\right) Q_{0}(t, x),
$$

for some distinct unit vectors $\widetilde{\nu}_{1}, \ldots, \widetilde{\nu}_{l}$ and some homogeneous polynomial $Q_{0}(x, t)$ of an even degree, with $Q_{0}(x, t) \neq 0$ for $(x, t) \neq 0$.

Suppose (7.3) holds. Then consider a unit vector $\eta$ orthogonal to $\nu$ and any function $u$ with small $|u|_{C^{1}}$. We have

$$
\partial_{\nu} K+u \partial_{\eta} K=-\partial_{\nu} P+R_{0} \equiv-\partial_{\nu} P+\left(-u \partial_{\eta} P+\partial_{\nu} R+u \partial_{\eta} R\right) .
$$

Compared with $\partial_{\nu} P, \partial_{\nu} R+u \partial_{\eta} R$ is a higher order term, and $-u \partial_{\eta} P$ is a small term, since $|u|_{C^{1}}$ is small. So we need to study the zero set of

$$
\left(\widetilde{\nu}_{1} \cdot(x, t)\right) \cdots\left(\widetilde{\nu}_{l} \cdot(x, t)\right) Q_{0}(t, x)-R_{0}(t, x)=0 .
$$

We can see easily that its zero set consists of $l$ curves through the origin and close to lines $\widetilde{\nu}_{i} \cdot(x, t)=0, i=1, \ldots, l$, and that across these curves the expression $\partial_{\nu} K+u \partial_{\mu} K$ changes its sign. To see this, we fix an $i$ and assume $\widetilde{\nu}_{i} \cdot(x, t)=c_{i}\left(t-a_{i} x\right)$ for some constants $c_{i} \neq 0$ and $a_{i}$. Set

$$
t=a_{i} x+x z(x) .
$$

Substitute in (7.4) and cancel $x^{n-1}$ from both side. We then use the implicit function theorem to get a solution $z(x)$ with small $C^{1}$-norm of $z$. This would finish the proof.

Note (7.3) means that $\partial_{\nu} P$ has no multiple linear factors. We allow irreducible quadratic factors to appear and even to have higher orders.

Next we prove (7.3). We introduce the polar coordinates $(x, t)=$ $(r \cos \theta, r \sin \theta)$. For the unit vector $\nu=(\cos \psi, \sin \psi)$, we have

$$
\begin{aligned}
\partial_{\nu} P & =\cos \psi \partial_{x} P+\sin \psi \partial_{t} P \\
& =\partial_{r} P(\cos \psi \cos \theta+\sin \psi \sin \theta)+\frac{1}{r} \partial_{\theta} P(-\cos \psi \sin \theta+\sin \psi \cos \theta) \\
& =\partial_{r} P \cos (\theta-\psi)-\frac{1}{r} \partial_{\theta} P \sin (\theta-\psi) .
\end{aligned}
$$


Suppose $P=r^{n} f(\theta)$ in polar coordinates. Then we get

$$
\partial_{\nu} P=r^{n-1}\left(n f(\theta) \cos (\theta-\psi)-f^{\prime}(\theta) \sin (\theta-\psi)\right) .
$$

Set

$$
g(\theta)=g(\theta ; \psi)=n f(\theta) \cos (\theta-\psi)-f^{\prime}(\theta) \sin (\theta-\psi) .
$$

Here we view $\psi$ as a parameter. Now we claim that by choosing $\psi$ appropriately the algebraic equation $g(\theta)=0$ does not have multiple roots.

To see this, we first calculate

$$
g^{\prime}(\theta)=(n-1) f^{\prime}(\theta) \cos (\theta-\psi)-\left(f^{\prime \prime}(\theta)+n f(\theta)\right) \sin (\theta-\psi) .
$$

Note (7.2) implies that if $f\left(\theta_{0}\right)=0$, then $f^{\prime}\left(\theta_{0}\right)=0$ and $f^{\prime \prime}\left(\theta_{0}\right) \neq 0$. Hence for such a $\theta_{0}, g\left(\theta_{0}\right)=0$ and

$$
g^{\prime}\left(\theta_{0}\right)=-f^{\prime \prime}\left(\theta_{0}\right) \sin \left(\theta_{0}-\psi\right)
$$

Therefore by choosing $\psi$ appropriately $\theta_{0}$ is not a double root of $g(\theta)=$ 0 . In fact there are only two choices of $\psi$ to make $\theta_{0}$ a double root. Now consider the case $g\left(\theta_{0}\right)=0$ and $f\left(\theta_{0}\right) \neq 0$. Then we have by $(7.5)$

$$
\cos \left(\theta_{0}-\psi\right)=\frac{f^{\prime}\left(\theta_{0}\right)}{n f\left(\theta_{0}\right)} \sin \left(\theta_{0}-\psi\right)
$$

Substituting (7.7) into (7.6) we get

$$
g^{\prime}\left(\theta_{0}\right)=\left((n-1) \frac{\left(f^{\prime}\left(\theta_{0}\right)\right)^{2}}{n f\left(\theta_{0}\right)}-f^{\prime \prime}\left(\theta_{0}\right)-n f\left(\theta_{0}\right)\right) \sin \left(\theta_{0}-\psi\right) .
$$

Note $\sin \left(\theta_{0}-\psi\right) \neq 0$. Otherwise $g\left(\theta_{0}\right)=n f\left(\theta_{0}\right) \cos \left(\theta_{0}-\psi\right) \neq 0$, which is a contradiction. Now we study the algebraic equation

$$
n f(\theta)\left(f^{\prime \prime}(\theta)+n f(\theta)\right)-(n-1)\left(f^{\prime}(\theta)\right)^{2}=0 .
$$

Equation (7.8) is independent of $\psi$. It is easy to see that the left side in (7.8) is not identically zero and hence (7.8) has at most finitely many roots $\theta_{1}, \ldots, \theta_{d}$. We may always choose $\psi$ so that $\theta_{1}, \ldots, \theta_{d}$ are not roots to

$$
\cos (\theta-\psi)=\frac{f^{\prime}(\theta)}{n f(\theta)} \sin (\theta-\psi)
$$


In other words, $\theta_{1}, \ldots, \theta_{d}$ are not roots to $g(\theta)=0$.

In conclusion, we have proved that by choosing $\psi$ appropriately if $g\left(\theta_{0}\right)=0$ then $g^{\prime}\left(\theta_{0}\right) \neq 0$. This finishes the proof. $\quad$ q.e.d.

Remark. An important case is given when the linear factors are not present, i.e., the leading polynomial $P(x, t)$ is a homogeneous polynomial such that $P(x, t)>0$ for $(x, t) \neq 0$. Another important case is when the leading polynomial $P$ is a homogeneous quadratic polynomial. This is because a nonnegative homogeneous quadratic polynomial is either the square of a linear function or an irreducible quadratic polynomial. The isometric embedding correspoding to the latter case was discussed by Nakamura [12].

\subsection{The Gaussian curvature vanishes at finite orders and leading polynomials are not decisive}

In some cases when $K$ vanishes at a finite order, we cannot simply look at the leading term. We need to consider the complete expression of $K$. This is the case when the leading polynomials have linear factors of order more than 2 .

Lemma 7.2. Suppose $K$ is given in a neighborhood $N$ of 0 by

$$
K=h(x, t) Q(x, t) \prod_{j=1}^{k}\left(\nu_{j} \cdot(x, t)-c_{j}(x, t)\right)^{2 n_{j}}
$$

where $h$ is a function with $h(0)<0, Q(x, t)$ is homogeneous polynomial with $Q(x, t)>0$ for $(x, t) \neq 0, n_{1}, \ldots, n_{k}$ are positive integers, $\nu_{1}, \ldots, \nu_{k}$ are distinct unit vectors and $c_{1}, \ldots, c_{k}$ are $C^{1}$ functions with $c_{j}(0)=0$ and $\nabla c_{j}(0)=0$. Then $K$ satisfies the assumption $(\mathrm{A})$.

The assumption (7.9) also has a simple geometric interpretation. The zero set of $K$ consists of finitely many curves nontangentially meeting at the origin and along each curve $K$ preserves its vanishing order except at the origin.

In (7.9), if $n_{1}=\cdots=n_{k}=1$, then $K$ is reduced to the case we just discussed, since it has the form given in (7.1).

The proof of Lemma 7.2 is similar to that of Lemma 7.1. For Lemma 7.1, we showed for some unit vector $\nu$ that the zero set of $\partial_{\nu} K$ consists of finitely many curves $\gamma_{i}, i=1, \ldots, l$, meeting at the origin nontangentially and that $\partial_{\nu} K$ vanishes along each curve $\gamma_{i}$ with the 
order one. In (7.9), if $n_{j} \geq 2$ for some $1 \leq j \leq k$, then clearly $\partial_{\nu} K$ vanishes with the multiple order along the curve

$$
\gamma_{j}=\left\{(x, t) ; \nu_{j} \cdot(x, t)-c_{j}(x, t)=0\right\},
$$

for any unit vector $\nu$. We will show by choosing $\nu$ appropriately, these are the only curves where $\partial_{\nu} K$ vanishes with multiple orders. On all other curves in its zero set, $\partial_{\nu} K$ vanishes with the order one.

Proof. Writing $b_{j}(x, t)=\nu_{j} \cdot(x, t)-c_{j}(x, t)$, we have

$$
K=h(x, t) Q(x, t) \prod_{j=1}^{k}\left(b_{j}(x, t)\right)^{2 n_{j}}
$$

Then for any pair of orthogonal unit vectors $(\eta, \nu)$, we get

$$
\begin{aligned}
& \partial_{\nu} K=h b_{1}^{2 n_{1}-1} \cdots b_{k}^{2 n_{k}-1}\left\{Q \left(2 n_{1} \hat{b}_{1} b_{2} \cdots b_{k} \partial_{\nu} b_{1}+\cdots\right.\right. \\
&\left.\left.+2 n_{k} b_{1} \cdots b_{k-1} \hat{b}_{k} \partial_{\nu} b_{k}\right)+\partial_{\nu} Q b_{1} b_{2} \cdots b_{k}+\frac{\partial_{\nu} h}{h} Q b_{1} b_{2} \cdots b_{k}\right\}
\end{aligned}
$$

where $\wedge$ indicates the corresponding expression is omitted. Set $l_{j}=$ $\nu_{j} \cdot(x, t)$ for $j=1, \ldots, k$. Inside the parenthesis $\{\cdot\}$ in $(7.10)$, the leading part is the homogeneous polynomial

$$
\widetilde{P}(x, t) \equiv Q\left(2 n_{1} \hat{l}_{1} l_{2} \cdots l_{k} \partial_{\nu} l_{1}+\cdots+2 n_{k} l_{1} \cdots l_{k-1} \hat{l}_{k} \partial_{\nu} l_{k}\right)+\partial_{\nu} Q l_{1} l_{2} \cdots l_{k}
$$

and the rest consists of higher order terms. Then for any function $u$ with small $|u|_{C^{1}}$, we have

$$
\partial_{\nu} K+u \partial_{\eta} K=\sigma b_{1}^{2 n_{1}-1} \cdots b_{k}^{2 n_{k}-1}\{\widetilde{P}+u \widetilde{\widetilde{P}}+\text { h.o.t. }\}
$$

where $\widetilde{\widetilde{P}}$ is obtained from $\widetilde{P}$ with $\partial_{\nu}$ replaced by $\partial_{\eta}$. Note each $b_{j}=0$ gives a curve through the origin tangent to the straight line $L_{i}$ given by $l_{i}=0$.

We claim that $\widetilde{P}$ does not have multiple linear factors and that $l_{1}, \ldots, l_{k}$ are not its linear factors by choosing $\nu$ appropriately. Then a perturbation argument as in the proof of Lemma 7.1 shows that $K$ satisfies (A). 
Now we prove the claim. First it is easy to see that $l_{i}$ is not a factor of $\widetilde{P}$ as long as $\nu$ is not orthogonal to the line $L_{i}$ generated by $l_{i}=0$. Since $\left.l_{i}\right|_{L_{i}}=0$, then

$$
\left.\widetilde{P}\right|_{L_{i}}=\left.2 n_{i}\left(Q l_{1} \cdots \hat{l}_{i} \cdots l_{k}\right)\right|_{L_{i}} \partial_{\nu} l_{i}
$$

which is not identically zero if $\nu$ is not orthogonal to $L_{i}$.

Next, we study zeros of $\widetilde{P}$. Since $\underline{i}_{\underset{P}{ }}$ cannot be factors for $\widetilde{P}$ by choosing $\nu$ appropriately, we may write $\widetilde{P}$ as

$$
\widetilde{P}=\frac{1}{l_{1}^{2 n_{1}-1} \cdots l_{k}^{2 n_{k}-1}} \partial_{\nu}\left(l_{1}^{2 n_{1}} \cdots l_{k}^{2 n_{k}} Q\right) .
$$

Set

$$
F=l_{1}^{2 n_{1}} \cdots l_{k}^{2 n_{k}} Q \text { and } F_{0}=l_{1}^{2 n_{1}-1} \cdots l_{k}^{2 n_{k}-1} .
$$

We then have

$$
\widetilde{P}=\frac{1}{F_{0}} \partial_{\nu} F
$$

We introduce the polar coordinates $(x, t)=(r \cos \theta, r \sin \theta)$. Set

$$
F=r^{n} f(\theta) \text { and } F_{0}=r^{m} f_{0}(\theta) .
$$

For the unit vector $\nu=(\cos \psi, \sin \psi)$, we then have as in the proof of Lemma 7.1

$$
\widetilde{P}=\frac{r^{n-m-1}}{f_{0}(\theta)}\left\{n f(\theta) \cos (\theta-\psi)-f^{\prime}(\theta) \sin (\theta-\psi)\right\} .
$$

Set

$$
g(\theta)=g(\theta ; \psi)=\frac{1}{f_{0}(\theta)}\left\{n f(\theta) \cos (\theta-\psi)-f^{\prime}(\theta) \sin (\theta-\psi)\right\} .
$$

Here we view $\psi$ as a parameter. To prove that $\widetilde{P}$ does not have multiple linear factors, we will show that the algebraic equation $g(\theta)=0$ does not have multiple roots.

By (7.11) and (7.12), we have

$$
f_{0}\left(\theta_{0}\right)=0 \text { if and only if } f\left(\theta_{0}\right)=0 .
$$

Hence $1 / f_{0}(\theta)$ is well-defined and not equal to zero for any $\theta$, which is not the root to $f(\theta)=0$. We already proved that the root $\theta_{0}$ to $f(\theta)=0$ 
cannot be the root to $g(\theta)=0$ by choosing $\psi$ appropriately. Hence we only need to discuss the case $g\left(\theta_{0}\right)=0$ and $f\left(\theta_{0}\right) \neq 0$. Proceed exactly as in the proof of Lemma 7.1, we conclude that by choosing $\psi$ further if $g\left(\theta_{0}\right)=0$ then $g^{\prime}\left(\theta_{0}\right) \neq 0$. This finishes the proof. $\quad$ q.e.d.

In a special case when $Q \equiv 1$ and $l=1$, the corresponding isometric embedding was discussed by Hong [5]. In this case the Gaussian curvature $K$ has the form

$$
K=h(x, t)(g(x, t))^{2 m},
$$

for some functions $h(x, t)$ and $g(x, t)$ with $h(0)<0, g(0)=0$ and $\nabla g(0) \neq 0$.

\subsection{The Gaussian curvature vanishes up to infinite orders}

The condition (A) also allows $K$ to vanish to infinite orders. In fact, if $\widetilde{K}$ satisfies (A) with $\widetilde{K} \leq 0$, then

$$
K=-\exp \left\{\frac{1}{\widetilde{K}}\right\}
$$

also satisfies (A).

\section{References}

[1] Y. Egorov, Sur un exemple d'equation lineaire hyperbolique n'ayant pas de solution, Journees "Équations aux Dérivées Partielles" (Saint-Jean-de-Monts, 1992), Exp. No. XIV, 6 pp., École Polytech., Palaiseau, 1992, MR 94b:35157, Zbl 0779.35070.

[2] K.O. Friedrichs, Symmetric positive linear differential equations, Comm. Pure Appl Math. 11 (1956) 333-418, MR 20 \#7147, Zbl 0083.31802.

[3] M. Gromov, Partial Differential Relations, Ergebnisse der Mathematik und ihrer Grenzgebiete, 9, Springer-Verlag, Berlin, 1986, MR 90a:58201, Zbl 0651.53001.

[4] M. Günther, Isometric embeddings of Riemannian manifolds, Proceedings of the International Congress of Mathematicians, Vol. II, (Kyoto 1990), Math. Soc. Japan, Tokyo, 1991, 1137-1143, MR 93b:53049, Zbl 0745.53031.

[5] J.-X. Hong, Cauchy problems for degenerate hyperbolic Monge-Ampère equations and some applications, J. Partial Diff. Equations 4 (1991) 1-18, MR 92d:35205, Zbl 0753.35055 . 
[6] L. Hörmander, On the Nash-Moser implicit function theorem, Ann. Acad. Sci. Fenn. Ser. A. I. Math. 10 (1985) 255-259, MR 87a:58025, Zbl 0591.58003.

[7] H. Jacobowitz, Local isometric embeddings, Seminar on Differential Geometry, Ann. of Math. Stud., 102, Princeton University Press, Princeton, 1982, 381393, MR 83g:53022, Zbl 0481.53018.

[8] C.-S. Lin, The local isometric embedding in $\mathbf{R}^{3}$ of 2-dimensional Riemannian manifolds with nonnegative curvature, J. Differential Geometry 21 (1985) 213-230, MR 87m:53073, Zbl 0584.53002.

[9] C.-S. Lin, The local isometric embedding in $\mathbf{R}^{3}$ of two-dimensional Riemannian manifolds with Gaussian curvature changing sign cleanly, Comm. Pure Appl. Math. 39 (1986) 867-887, MR 88e:53097, Zbl 0612.53013.

[10] N. Nadirashvili, The local embedding problems for surfaces, preprint, 2002.

[11] N. Nadirashvili \& Y. Yuan, Counterexamples for local isometric embedding, preprint, 2002.

[12] G. Nakamura, Local isometric embedding of two-dimensional Riemannian manifolds into $\mathbf{R}^{\mathbf{3}}$ with nonpositive Gaussian curvature, Tokyo J. Math. 10 (1987) 241-257, MR 89b:53008, MR 0628.53023.

[13] G. Nakamura \& Y. Maeda, Local smooth isometric embeddings of low dimensional Riemannian manifolds into Euclidean spaces, Trans. Amer. Math. Soc. 313 (1989) 1-51, MR 90f:58171, Zbl 0682.58046.

[14] J. Nash, The imbedding problem for Riemannian manifolds, Annals of Math. 63 (1956) 20-63, MR 17,782b, Zbl 0070.38603.

[15] E.G. Poznyak \& E.V. Shikin, Small parameters in the theory of isometric imbeddings of two-dimensional Riemannian manifolds in Euclidean spaces, Some questions of differential geometry in the large (E.V. Shikin, ed.), Amer. Math. Soc. Transl. (2) 176 (1996) 151-192, MR 98c:53006, Zbl 0862.53004.

[16] B. Rozhdestvenskiı̆, Quasilinear hyperbolic systems for theory of surfaces, Dokl. Akad. Nauk. USSR 143 (1962) 50-52.

[17] S.-T. Yau, Problem section, Seminar on Differential Geometry, Ann. of Math. Stud., 102, Princeton University Press, Princeton, 1982, 669-706, MR 83e:53029, Zbl 0479.53001.

[18] S.-T. Yau, Open problems in geometry, Chern - a great geometer of the twentieth century, Internat. Press, Hong Kong, 1992, 275-319, MR 1201369.

Department of MATHEMatics University of Notre Dame, Notre Dame, IN 46556

and

MaX-Planck Institute for Mathematics 
INSELSTR. $22-26$ 04103 Leipzig, Germany

Institute of MATHEMATiCS Fudan University, Shanghai, China Department of Mathematics National Chung-Cheng University Ming-Hsiung, Chiayi, Taiwan 